\title{
Spatial Verification of Ensemble Precipitation: An Ensemble Version of SAL
}

\author{
SABINE RADANOVICS \\ CNRM UMR 3589, Météo-France/CNRS, Toulouse, France \\ JEAN-PHILIPPE VIDAL AND ERIC SAUQUET \\ Irstea, UR RiverLy, Centre de Lyon-Villeurbanne, Villeurbanne, France
}

(Manuscript received 6 November 2017, in final form 7 May 2018)

\begin{abstract}
Spatial verification methods able to handle high-resolution ensemble forecasts and analysis ensembles are increasingly required because of the increasing development of such ensembles. An ensemble extension of the structure-amplitude-location (SAL) spatial verification method is proposed here. The ensemble SAL (eSAL) allows for verifying ensemble forecasts against a deterministic or ensemble analysis. The eSAL components are equal to those of SAL in the deterministic case, thus allowing the comparison of deterministic and ensemble forecasts. The Mesoscale Verification Intercomparison over Complex Terrain (MesoVICT) project provides a dataset containing deterministic and ensemble precipitation forecasts as well as a deterministic and ensemble analysis for case studies in summer 2007 over the greater Alpine region. These datasets allow for testing of the sensitivity of SAL and eSAL to analysis uncertainty and their suitability for the verification of ensemble forecasts. Their sensitivity with respect to the main parameter of this featurebased method-the threshold for defining precipitation features-is furthermore tested for both the deterministic and ensemble forecasts. Our results stress the importance of using meaningful thresholds in order to limit any unstable behavior of the threshold-dependent SAL components. The eSAL components are typically close to the median of the distribution of deterministic SAL components calculated for all combinations of ensemble members of the forecast and the analysis, with considerably less computational time. The eSAL ensemble extension of SAL can be considered as a relevant summary measure that leads to more easily interpretable SAL diagrams.
\end{abstract}

\section{Introduction}

Spatial verification techniques have been developed within the context of quantitative precipitation forecasting with high-resolution numerical weather forecast models. The developments were motivated by studies such as that by Mass et al. (2002), whose results revealed that despite high-resolution forecasts showing more realistic structures, traditional point verification scores may suggest low skill especially in the presence of timing or position errors. Scale separation (Briggs and Levine 1997; Denis et al. 2002; Casati et al. 2004; Yano and Jakubiak 2016; Weniger et al. 2017) and neighborhood methods (Zepeda-Arce et al. 2000; Roberts and Lean 2008; Ebert 2008; Mittermaier 2014; Skok and Roberts 2016) are useful in determining skillful scales for example. However, such

Corresponding author: Sabine Radanovics, sabine.radanovics@ meteo.fr methods do not treat displacement and structure errors explicitly. Deformation methods (Gilleland et al. 2010; Keil and Craig 2007; Gilleland 2011; Farchi et al. 2016) have strong capacities in describing displacement errors, while object-based methods (AghaKouchak et al. 2011; Ebert and McBride 2000; Davis et al. 2006, 2009; Griffin et al. 2017; Mittermaier et al. 2016; Wernli et al. 2008) can take into account displacement and spatial structure errors at a given scale. A first method intercomparison project was started in 2007 using geometric cases and deterministic precipitation forecasts with prescribed errors over the U.S. Great Plains to assess the behavior of the different methods [see Gilleland et al. (2009) for results].

The second intercomparison project, called Mesoscale Verification Intercomparison over Complex Terrain (MesoVICT) (Dorninger et al. 2013), focused on convective precipitation cases during summer 2007 over a region with complex terrain. In addition the project aimed at exploring how the different methods can cope 
with the verification of ensemble forecasts, how they can account for observation uncertainties, and how sensitive they are to parameter settings (Dorninger et al. 2013). Some methods including the structure-amplitude-location (SAL) method (Wernli et al. 2008) have already been tested on the MesoVICT cases (Dorninger and Gorgas 2013; Geiß 2015; Gilleland 2017) but work that addresses the spatial verification of ensemble forecasts is still rare.

So far, only a few spatial verification measures have been developed for assessing ensemble simulations (Ben Bouallègue and Theis 2014; Duc et al. 2013), most of them neighborhood methods (Dey et al. 2014; Marsigli et al. 2008; Theis et al. 2005; Schwartz and Sobash 2017), or methods used to assess differences between ensemble members or ensemble spread (Keil and Craig 2007; Barrett et al. 2015; Dey et al. 2016). Exceptions include the work of Gallus (2010), who averaged feature characteristics obtained with the contiguous rain area (CRA; Ebert and McBride 2000) and Method for Object-Based Diagnostic Evaluation (MODE; Davis et al. 2006, 2009) methods over all ensemble members containing a matched feature. A similar approach was proposed by Skinner et al. (2016) for ensemble forecasts of mesocyclones. Further, Fox et al. (2016) used Bayesian Procrustes shape analysis to define meaningful ensemble mean features, Schwartz et al. (2017) compared locations of probability objects with locations of observed objects, and Mittermaier and Bullock (2013) and later Clark et al. (2014) extended MODE to three-dimensional objects where the third dimension is the time dimension. In principle the third dimension could probably be an ensemble dimension as well, but published research on this subject is still lacking.

In parallel, the role of observation uncertainty in forecast verification has gained attention during the last few years (e.g., Ebert et al. 2013; Mittermaier and Stephenson 2015) since it can no longer be neglected as high-resolution short-term forecasts become more accurate. Concerning the observation uncertainty, Röpnack et al. (2013) used Bayes's factors to verify and compare different ensemble forecasts. The logarithm of the Bayes factor was shown to be equivalent to the ignorance score (Good 1952; Roulston and Smith 2002) but taking the observation uncertainty and the spatial correlation structure into account. Bowler $(2006,2007)$ used the observation-error estimates from a data assimilation procedure and proposed observation-errorcorrected versions of the RMSE, rank histograms, and contingency table based scores. Candille and Talagrand (2008) presented a version of the Brier score and the relative operating characteristics (ROC) area score for noisy observations. Observation uncertainties can also be expressed via analysis ensembles such as the radar ensemble generator designed for usage in the Alps using a lower-upper (LU) decomposition (REAL) dataset (Germann et al. 2009), analysis ensembles obtained from ensemble data assimilation (Lange and Craig 2014), or the Vienna Enhanced Resolution Analysis (VERA) ensemble available for the MesoVICT case 1 (Gorgas and Dorninger 2012a). The ultimate goal of using observation or analysis ensembles is to provide confidence intervals for verification measures (Gorgas and Dorninger 2012b), but to this end the behavior of the verification procedures with respect to the observation uncertainty has to be explored first. Weniger and Friederichs (2016) argued that threshold sensitivity can be used as a proxy for the behavior of SAL with respect to observation uncertainty. However, the VERA ensemble offers the possibility of assessing the effects of the observation uncertainty more directly with more realistic variations.

The objective of this paper is therefore twofold: first, an ensemble version of the SAL method initially proposed by Wernli et al. (2008) is introduced. The ensemble version of SAL (eSAL) is then applied to the ensemble forecasts from the MesoVICT project (Dorninger et al. 2013) and compared with results for the deterministic SAL. Second, the sensitivity of SAL and eSAL to analysis uncertainty is explored using the threshold sensitivity method (Weniger and Friederichs 2016) as well as through a direct assessment with the VERA ensemble as reference.

The data and the study area are presented in section 2 . SAL and the developed extensions of the method are detailed in section 3 . Section 4 gives a brief overview over the experiments whose results are shown in section 5. Issues concerning threshold sensitivity, observation uncertainty, and the verification of probability fields are discussed in section 6 , and conclusions are given in section 7 .

\section{Data and study area}

During the Convective and Orographically Induced Precipitation Study (COPS) experiment (Wulfmeyer et al. 2008), a wide range of observation, analysis, and forecast data were collected over the study area centered over the Black Forest region in southern Germany (Fig. 1). For the MesoVICT project, six case studies, spanning a total of 18 days between June and September 2007 , have been selected. In this study we focus on case 1, 20-22 June 2007, because this case is the only one for which the ensemble forecasts are available. However, for the sensitivity experiment using only deterministic forecast and analysis datasets, data from the other five cases (8-10 and 18-21 July, 6-8 August, and 18 and 25-28 September 2007) are used as well in order to obtain more robust results. 


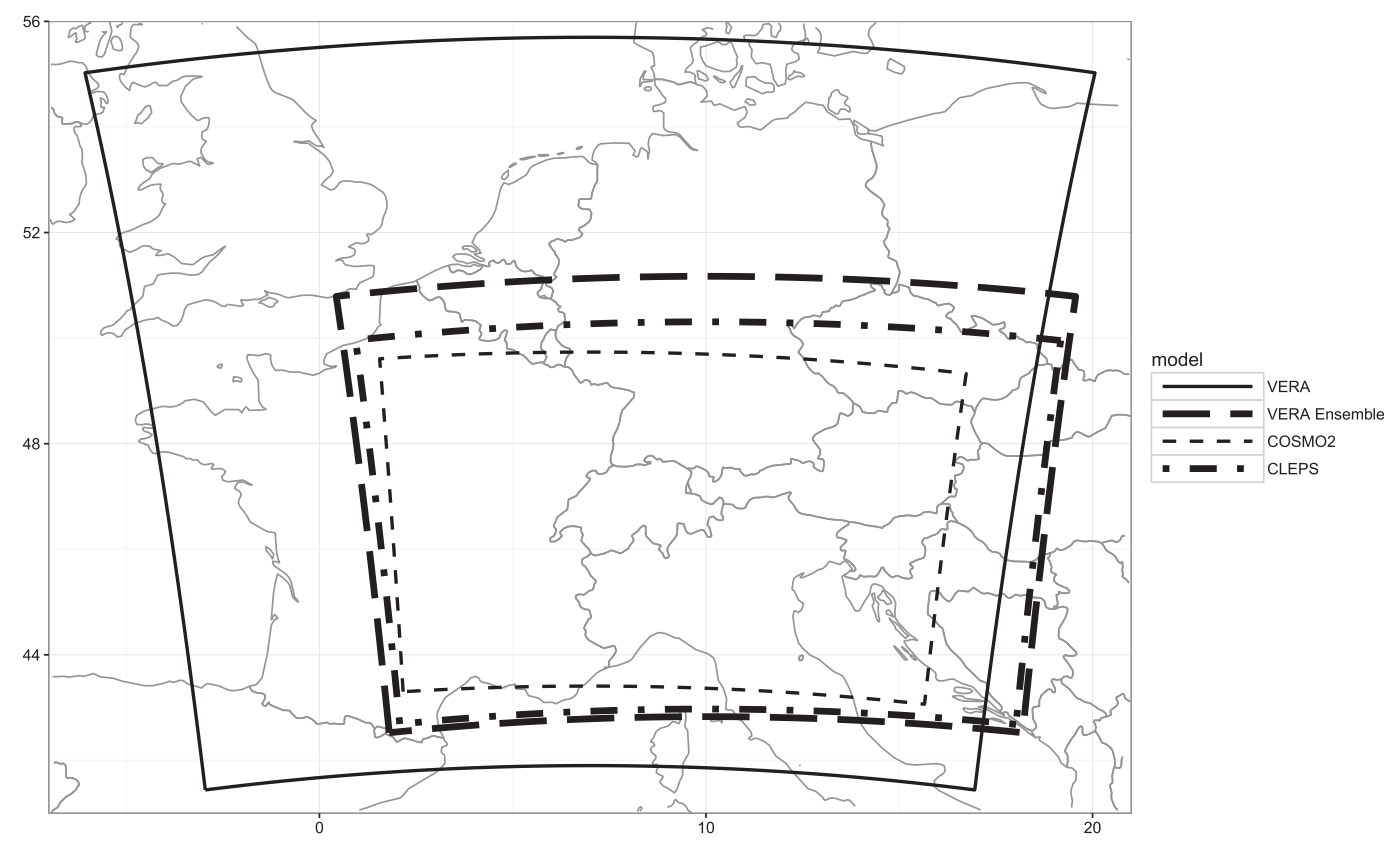

FIG. 1. Spatial domains over which the datasets used are available.

The following analysis and forecast datasets are used in the present study.

VERA hourly precipitation analysis (Steinacker et al. 2000) for the six case studies and 3-hourly precipitation analysis for case 1: The VERA analysis is based on thin-plate spline interpolation of irregularly distributed observations on an $8-\mathrm{km}$ grid. Further, it makes use of a priori knowledge about small-scale structures due to topography or dynamic processes, which allows adding small-scale features that are not explicitly resolved by the observations (Steinacker et al. 2006; Bica et al. 2007). A quality control scheme helps avoid artificial patterns due to gross errors (Steinacker et al. 2011).

COSMO2 (Baldauf et al. 2011; Ament and Arpagaus 2009) deterministic hourly precipitation forecasts for the six case studies and 3-hourly precipitation forecasts for case 1: The forecasts were initialized each day at 0000 UTC with lead times up to $24 \mathrm{~h}$. The original resolution is $2.2 \mathrm{~km}$, but for MesoVICT the model fields were interpolated onto the $8-\mathrm{km}$ VERA grid. The model is convection permitting (i.e., deep convection is not parameterized).

VERA hourly 50-member ensemble precipitation analysis for case 1 (Gorgas and Dorninger 2012a): Unlike probabilistic interpolation methods such as kriging, the deterministic interpolation used in VERA does not directly give uncertainty estimates. Therefore, Gorgas and Dorninger (2012a) created perturbations of the observations using residuals from the quality control procedure as uncertainty estimates. The perturbed observations were then interpolated to create the 50 realizations of the analysis, using again the VERA quality control scheme to remove gross errors and inconsistencies (Gorgas and Dorninger 2012a). A 2D-wavelet decomposition is used to find the structures containing the highest wave energy, and the wavelet coefficients are used as weights in order to locate the highest perturbations around the main precipitation events (Gorgas and Dorninger 2012a). We built 3-hourly sums of the VERA ensemble members for a direct comparison with the COSMO Limited-Area Ensemble Prediction System (CLEPS) forecasts (see next paragraph). Because of the missing temporal coherence of the perturbations, the ensemble variance of the 3-hourly data may be too small. We performed a set of tests to see if the 3-hourly sums led to a reasonable ensemble. As for the 1-hourly ensemble, the spatial variance of the ensemble members tends to be distributed around or slightly above the deterministic one, as expected because of the added perturbations. Based on our tests we concluded that even though the analysis ensemble based on 3-hourly sums might not correctly represent the analysis uncertainty, it has enough variance to provide a meaningful test bed for the behavior of SAL with respect to analysis uncertainty.

CLEPS 3-hourly 16-member ensemble precipitation forecasts for case 1 (Montani et al. 2003): The CLEPS 
forecasts were initialized each day at 1200 UTC with lead times up to $132 \mathrm{~h}$. In this study we use the forecasts with lead times between 3 and $120 \mathrm{~h}$ (5 days), which gives five forecasts with different lead times for each verification time step. CLEPS has an original resolution of $10 \mathrm{~km}$ and was interpolated onto the 8-km VERA ensemble grid. CLEPS is based on the COSMO-10 model and uses 16 members of the ECMWF Ensemble Prediction System (ECMWF-EPS) as boundary conditions (Marsigli et al. 2005). In addition the model physics are perturbed, namely the convection scheme (Kain-Fritsch or Tiedtke schemes are selected randomly for the different members), the maximal turbulent length scale, and the length scale of subscale surface patterns (Röpnack et al. 2013).

The respective model domains are shown in Fig. 1. The verification is always performed on the COSMO2 domain.

\section{Structure-amplitude-location: SAL}

\section{a. Standard $S A L$}

SAL is a spatial verification method developed by Wernli et al. (2008) for the verification of highresolution NWP precipitation forecasts. The method compares three characteristics of observed and simulated precipitation: 1) the amplitude $A$, that is, the total precipitation over the domain; 2) the location $L$, that is, where precipitation is located; and 3) the structure $S$, that is, the size and the shape of precipitation objects. Precipitation objects, also called features, are contiguous locations where precipitation is above a given threshold. The original formulas from Wernli et al. (2008) are reproduced below for reference. The amplitude is

$$
A=\frac{\overline{\mathrm{rr}_{\text {mod }}}-\overline{\mathrm{rr}_{\mathrm{obs}}}}{0.5\left(\overline{\mathrm{rr}_{\mathrm{mod}}}+\overline{\mathrm{rr}_{\mathrm{obs}}}\right)},
$$

where $\overline{\mathrm{rr}}$ is the domain average precipitation, and the mod and obs subscripts refer to modeled and observed precipitation, respectively. The value of $A$ ranges from -2 to 2 , where perfect agreement of the domain average precipitation results in $A=0$, too little modeled precipitation results in $A<0$, and too high modeled precipitation gives $A>0$. Next, the expressions for location are as follows:

$$
L_{1}=\frac{\left|\mathbf{x}\left(\mathrm{rr}_{\bmod }\right)-\mathbf{x}\left(\mathrm{rr}_{\mathrm{obs}}\right)\right|}{d}
$$

where $\mathbf{x}$ denotes the center of mass of the precipitation field, and $d$ is the largest distance between two domain borders. The value of $L_{1}$ ranges from 0 to 1 , where perfect agreement of the center of mass leads to $L_{1}=0$, and centers of mass located at the opposite domain border would lead to $L_{1}$ close to 1 . Next,

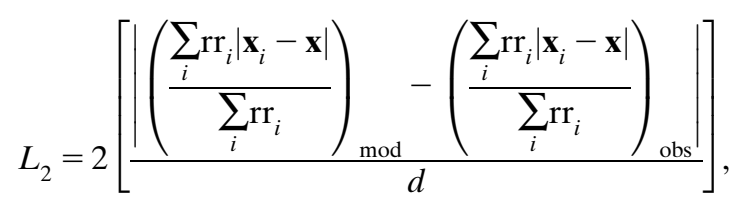

where $\mathbf{x}_{i}$ is the center of mass of the $i$ th feature, and $\mathrm{rr}_{i}$ is the sum of precipitation over all grid cells in feature $i$. Note that the features are not matched between the forecast and the observation, and therefore the number of features in each of the fields may differ. Like $L_{1}$, $L_{2}$ ranges from 0 to 1 . If there is only one feature in each field, then $L_{2}=0$. If the weighted average distance of the features to the global center of mass is similar in both fields, $L_{2}$ is small (close to zero); for example, all features are concentrated within a small portion of the domain in both the forecast field and the observation field, or the features are far from each other in both the forecast field and the observation field. If the features are close together in one field (forecast or observation) while the features are far from each other in the other field, $L_{2}$ is large (close to 1). Finally,

$$
L=L_{1}+L_{2}
$$

The value of $L$ ranges from 0 to 2 and is zero for a perfect forecast in terms of location. The structure component is

$$
S=\frac{V_{\text {mod }}-V_{\text {obs }}}{0.5\left(V_{\text {mod }}+V_{\text {obs }}\right)},
$$

where

$$
V=\frac{\sum_{i}\left(\operatorname{rr}_{i} \frac{\mathrm{rr}_{i}}{\operatorname{rr}_{i}^{\text {max }}}\right)}{\sum_{i} \mathrm{rr}_{i}},
$$

and $\mathrm{rr}_{i}$ is the sum of precipitation over all grid cells in feature $i$, and $\operatorname{rr}_{i}^{\max }$ is the maximum precipitation of the $i$ th feature. The value of $S$ ranges from -2 to 2 , where $S=0$ corresponds to equal average scaled volumes in the forecast and the observation, $S<0$ for too small or too peaked forecasted features, and $S>0$ for too large or too flat forecasted features.

SAL is not defined if there is no precipitation observed and/or no precipitation forecasted over the verification domain. As a consequence, SAL is a quality measure suited 
for case studies, but SAL is unable to properly account for missed events or false alarms. Time averages over SAL components are therefore not meaningful. Note that there is always some precipitation in the verification domain for the MesoVICT case study used in this paper. Unlike methods like the displacement and amplitude score (Keil and Craig 2009), SAL does not attempt to match individual features. The difference between the $A$ component of SAL and error measures like the mean absolute error (MAE) is that $A$ is a relative measure and the MAE an absolute one. Therefore, if the forecasted precipitation over the verification domain is half the observed one, $A$ will always have a value of -0.66 regardless of the absolute amounts, while the MAE will be higher for cases with higher precipitation amounts. The structure component rates the ability of the forecast to produce precipitation features with similar size and shape characteristics in terms of scaled volumes. The structure component aims to reward forecasts that show, for example, small and peaked precipitation objects, typical of convective precipitation, in case such precipitation is observed.

We use the $\mathrm{R}$ package SpatialVx version 0.5 (Gilleland 2016) for the SAL calculations with two modifications concerning the $L_{2}$ component whose implementation in SpatialVx version 0.5 is slightly different from the original SAL formulation: 1) we calculate all feature centers of mass using the intensity fields rather than the feature masks (the masks being fields containing 1 for grid cells belonging to a feature and 0 where there is no feature), and 2) we calculate the distance between the individual feature centroids and the whole-field centroid calculated for the $L_{1}$ component rather than the distance to the centroid of the feature masks.

\section{b. Enhanced SAL diagrams}

SAL diagrams were introduced by Wernli et al. (2008) to summarize the SAL components over a large number of time steps in one diagram. Each forecast is represented by a point located corresponding to its $S$ ( $x$ axis) and $A$ ( $y$ axis) components and is colored corresponding to the $L$ component. Points representing good forecasts are therefore situated near the center of the diagram and colored in light colors. We show an enhanced version of SAL diagrams where the point size is proportional to the mean nonzero precipitation over the reference field. These diagrams are useful for diagnosing if there are errors that are specific to cases with little precipitation or with high precipitation amounts in the domain.

\section{c. A proposed extension of $S A L$ for ensemble forecasts: eSAL}

SAL has so far mainly been used in high-resolution NWP model development (Ahijevych et al. 2009;
Zappa et al. 2010; Van Weverberg et al. 2010; Wittmann et al. 2010; Termonia et al. 2011; Haiden et al. 2011), evaluation (Zimmer et al. 2011; Hardy et al. 2016; Vincendon et al. 2011; Gofa et al. 2017), or model intercomparison (Zimmer 2010) for the verification of deterministic forecasts. For its application on ensemble forecasts there are two main possibilities: 1) the ensemble is treated as a collection of deterministic forecasts and distributions of SAL components are analyzed, or 2) the ensemble is verified as a whole, and thus the quality measures describe the performance of the ensemble as a whole. Examples of the first case can be found in Hanley et al. (2013) and Lange and Craig (2014).

Here, we propose a way to characterize the performance of the ensemble as a whole. The formulas are given for the case where the forecast and the analysis are both ensembles, but the formulas can easily be adapted to the case where only one of them is an ensemble. In the case of a deterministic forecast and a deterministic analysis, they are equal to the original SAL.

For $e A$ we simply added an ensemble average \langle\rangle to the original formula:

$$
e A=\frac{\left\langle\overline{\mathrm{rr}_{\mathrm{mod}}}\right\rangle-\left\langle\overline{\mathrm{rr}_{\mathrm{obs}}}\right\rangle}{0.5\left(\left\langle\overline{\mathrm{rr}_{\mathrm{mod}}}\right\rangle+\left\langle\overline{\mathrm{rr}_{\mathrm{obs}}}\right\rangle\right)},
$$

such that $e A$ is the relative difference of the ensemble average of the domain average precipitation in the forecast and the analysis. Note that the spatial average is applied before the ensemble average. Similarly, for $e L_{1}$, which becomes the absolute difference of the ensemble averages of the centers of mass divided by the maximum domain dimension $d$,

$$
e L_{1}=\frac{\left|\left\langle\mathbf{x}\left(\mathrm{rr}_{\text {mod }}\right)\right\rangle-\left\langle\mathbf{x}\left(\mathrm{rr}_{\text {obs }}\right)\right\rangle\right|}{d},
$$

where $\mathbf{x}(\mathrm{rr})$ denotes the center of mass of all precipitation in the field of an ensemble member. The ensemble average is thus applied to the coordinates of the center of mass in each individual field. We defined $e L_{2}$ as 2 times the continuous ranked probability score (CRPS) of the weighted average distances between the center of mass of an individual object and the total center of mass of the field divided by the maximum domain dimension d. The CRPS (Brown 1974; Matheson and Winkler 1976) is a probabilistic verification score that is widely used for the verification of ensemble forecasts (see, e.g., Hagedorn et al. 2008; Demargne et al. 2010; Aspelien et al. 2011; Wang et al. 2012). Recently, Goessling and Jung (2018) introduced the spatial probability score (SPS), which is defined as a spatial integral of local Brier scores and can be considered as a spatial version of the CRPS for the verification of probabilistic contours. 
The CRPS is a distance between two cumulative distribution functions, and it is equivalent to the absolute difference in the case of two-step functions, that is, in the deterministic forecast and deterministic analysis case (Hersbach 2000). The equivalence with the MAE in the purely deterministic case is critical for comparison with the standard SAL since the CRPS replaces the absolute difference in the $L_{2}$ formulation. The CRPS is computed as

$$
\operatorname{CRPS}\left(P_{f}, P_{a}\right)=\int_{-\infty}^{\infty}\left[P_{f}(x)-P_{a}(x)\right]^{2} d x,
$$

where $P_{f}$ and $P_{a}$ are the empirical cumulative distribution functions of the forecast and the analysis or observation, respectively. The properties of the CRPS are as described in Hersbach (2000). The CRPS is sensitive to the entire range of the simulated variable; that is, it evaluates the entire probabilistic prediction and no predefined classes are required, and it is equal to the MAE in the case of a deterministic forecast. The CRPS can be interpreted as an integral over all possible Brier scores, and it is a proper score (Gneiting and Raftery 2007). A proper score is one that encourages the forecaster to issue forecasts corresponding to his or her best judgment, because the score takes its ideal value only for ideal forecasts (Murphy 1969). Thus, $e L_{2}$ can be written as

$$
e L_{2}=2 \times \operatorname{CRPS}\left[P\left(\frac{r_{\mathrm{mod}}}{d}\right), P\left(\frac{r_{\mathrm{obs}}}{d}\right)\right],
$$

where

$$
r=\frac{\sum_{i} \mathrm{rr}_{i}\left|\mathbf{x}_{i}-\mathbf{x}\right|}{\sum_{i} \mathrm{rr}_{i}},
$$

and $\mathbf{x}_{i}$ is the center of mass of the $i$ th feature, $\mathrm{rr}_{i}$ is the sum of precipitation over all grid cells in feature $i$, and $P(r / d)$ is the empirical cumulative distribution function of $r / d$ of all ensemble members. Again, $d$ is the largest distance between two domain borders. The total location component is then

$$
e L=e L_{1}+e L_{2} .
$$

Note that even though the basic CRPS formula describes a difference between two distributions, common implementations of the CRPS, like in the R packages verification (Gilleland 2015), SpecsVerification (Siegert 2017), or scoringRules (Jordan et al. 2017), take either one ensemble and a single observation per time step or forecast distribution parameters (e.g., a mean and a standard deviation) and a single observation per time step and are not designed to work with two ensembles, that is, a forecast ensemble and an observation ensemble. Therefore, we use our own CRPS implementation. Schematic diagrams detailing the $e L$ calculation can be found in the appendix.

The structure component is equivalent to the deterministic version but for ensemble averages of the scaled volumes from each member:

$$
e S=\frac{\left\langle V_{\text {mod }}\right\rangle-\left\langle V_{\text {obs }}\right\rangle}{0.5\left(\left\langle V_{\text {mod }}\right\rangle+\left\langle V_{\text {obs }}\right\rangle\right)},
$$

where

$$
V=\frac{\sum_{i}\left(\mathrm{rr}_{i} \frac{\mathrm{rr}_{i}}{\operatorname{mr}_{i}^{\max }}\right)}{\sum_{i} \mathrm{rr}_{i}},
$$

and $\mathrm{rr}_{i}$ is the sum of precipitation over all grid cells in feature $i$, and $\operatorname{rr}_{i}^{\max }$ is the maximum precipitation of the $i$ th feature. First, the scaled volumes are calculated for each feature of each ensemble member, second the weighted average scaled volume is calculated within each ensemble member, third the ensemble average is applied to the weighted average scaled volumes, and finally the relative difference between the forecast and observations in terms of scaled volumes is calculated. Here, $e S$ refers to the ability of the ensemble as a whole to simulate, on average, the right scaled volumes. The idea of using an ensemble average of feature characteristics is similar to the approaches used by Skinner et al. (2016) or Gallus (2010). We tested an alternative definition of $e S$, taking the CRPS between distributions of scaled volumes, but this approach has the disadvantage of losing information on the sign, that is, if the simulated features are too small or too large, and also losing the equivalence with the standard SAL for purely deterministic cases.

Like SAL, eSAL is not defined if there is either no precipitation in the observation fields or no precipitation in the forecast fields. For eSAL to be calculated there has to be at least one ensemble member containing precipitation in the forecast and at least one ensemble member containing precipitation in the observation. The ensemble averages and the CRPS for the $e L$ and $e S$ components are calculated over the members containing precipitation. The $e A$ component is calculated over all ensemble members including the ones with zero precipitation.

\section{Experiments}

We conduct a series of experiments designed to demonstrate the behavior of SAL and eSAL. The experiments are briefly described in the following subsections and summarized in Table 1. 
TABLE 1. List of experiments.

\begin{tabular}{|c|c|c|c|c|c|c|c|c|}
\hline No. & Type & Experiment & Forecast & "Truth" & $\begin{array}{l}\text { Quality } \\
\text { measure }\end{array}$ & $\begin{array}{l}\text { Time } \\
\text { step }\end{array}$ & $\begin{array}{l}\text { Lead-time } \\
\text { class (days) }\end{array}$ & Case \\
\hline 1 & $\begin{array}{l}\text { Threshold } \\
\text { sensitivity }\end{array}$ & $\begin{array}{l}\text { Threshold sensitivity } \\
\text { SAL }\end{array}$ & COSMO2 & VERA & SAL & Hourly & 1 & $1-6$ \\
\hline 2 & $\begin{array}{l}\text { Threshold } \\
\text { sensitivity }\end{array}$ & $\begin{array}{l}\text { Threshold sensitivity } \\
\text { eSAL }\end{array}$ & COSMO2 & $\begin{array}{l}\text { VERA } \\
\text { ensemble }\end{array}$ & eSAL & Hourly & 1 & 1 \\
\hline 3 & Intraensemble & eSAL for similar fields & $\begin{array}{l}\text { CLEPS } \\
\text { members 2-16 }\end{array}$ & $\begin{array}{l}\text { CLEPS } \\
\text { member } 1\end{array}$ & eSAL & 3-hourly & 1 & 1 \\
\hline 4 & Intra-analysis & $\begin{array}{l}\text { VERA ensemble } \\
\text { compared to VERA }\end{array}$ & $\begin{array}{l}\text { VERA } \\
\text { ensemble }\end{array}$ & VERA & eSAL & $\begin{array}{l}\text { Hourly and } \\
\text { 3-hourly }\end{array}$ & - & 1 \\
\hline 5 & $\begin{array}{l}\text { Forecast } \\
\quad \text { verification }\end{array}$ & $\begin{array}{l}\text { SAL distributions due to } \\
\text { analysis uncertainty }\end{array}$ & COSMO2 & $\begin{array}{l}\text { VERA } \\
\text { ensemble }\end{array}$ & $\begin{array}{l}\text { SAL and } \\
\text { eSAL }\end{array}$ & Hourly & 1 & 1 \\
\hline 6 & $\begin{array}{l}\text { Forecast } \\
\quad \text { verification }\end{array}$ & $\begin{array}{l}\text { Verify ensemble forecasts } \\
\text { SAL vs eSAL }\end{array}$ & CLEPS & VERA & $\begin{array}{l}\text { SAL and } \\
\text { eSAL }\end{array}$ & 3-hourly & $1-5$ & 1 \\
\hline 7 & $\begin{array}{l}\text { Forecast } \\
\quad \text { verification }\end{array}$ & Compare models & $\begin{array}{l}\text { COSMO2 and } \\
\text { CLEPS }\end{array}$ & $\begin{array}{l}\text { VERA and } \\
\text { VERA ensemble }\end{array}$ & eSAL & 3-hourly & 1 & 1 \\
\hline
\end{tabular}

\section{a. Threshold sensitivity}

The threshold for defining objects had been subjectively defined in the early applications of the standard SAL method, as $R_{\max } \times f$ [Wernli et al. (2008) used $f=1 / 15]$, where $R_{\max }$ is the maximum precipitation value over the domain, or $R^{95} \times f$, where $R^{95}$ is the " 95 th percentile of all gridpoint values in the domain larger than $0.1 \mathrm{~mm}$ " (Wernli et al. 2009).

We conduct threshold sensitivity experiments where the threshold factor $f$ is varied. In contrast to Weniger and Friederichs (2016), who did similar experiments, we make sure to avoid meaningless thresholds, for example below or equal to the minimum value of the field, and absolute threshold differences smaller than the resolution of the data.

In experiment 1 we use the hourly deterministic precipitation forecast from COSMO2 for all 18 case study days and VERA hourly deterministic analysis over the COSMO 2 domain, resulting in a total of 432 cases. The lead times range from 0 to $23 \mathrm{~h}$ depending on the time of day. We choose independent thresholds for the forecast and the analysis field taking $R^{95} \times f$ of each field as the threshold, as in Wernli et al. (2009). For the sensitivity experiment, $f$ ranges from a minimum factor $f_{\min }$ to 1 with steps of $f_{\min }$. We calculate $f_{\min }$ as

$$
f_{\min }=\max \left(0.01, \frac{0.01 \mathrm{~mm}}{R_{\mathrm{mod}}^{95}}, \frac{0.01 \mathrm{~mm}}{R_{\mathrm{obs}}^{95}}\right),
$$

where 0.01 is a minimum value for $f_{\min }, 0.01 \mathrm{~mm}$ is the minimum nonzero value in the data, $R_{\bmod }^{95}$ is the 95 th percentile of the gridpoint values $>0$ in the forecast field, and $R_{\mathrm{obs}}^{95}$ is the 95th percentile of the gridpoint values $>0$ in the analysis field. The components that depend on the threshold are $S$ and $L_{2}$. If the SAL components were robust with respect to small changes in the precipitation fields, they should be robust with respect to small changes in the threshold as well. Therefore, the differences in the $S$ and $L_{2}$ components using two different threshold factors should tend toward 0 for very small threshold factor differences.

In experiment 2 we test the threshold sensitivity of eSAL. We use the hourly VERA ensemble analysis and the COSMO2 forecasts for the 3 days of case study 1 . There are in total 72 cases. The ensemble datasets (CLEPS and VERA ensemble) are only available for case 1 . We decided to take COSMO2 and the VERA ensemble for this experiment rather than CLEPS, because CLEPS has only 3-hourly forecasts and therefore even fewer cases. The thresholds and threshold factors are calculated the same way as in experiment 1 . Note that the same threshold is used for all ensemble members. Therefore, $R^{95}$ is calculated by pooling all gridpoint values $>0$ of all ensemble members.

For all other experiments below, $f$ is set to

$$
f=\max \left(\frac{1}{15}, \frac{0.01 \mathrm{~mm}}{R_{\mathrm{mod}}^{95}}, \frac{0.01 \mathrm{~mm}}{R_{\mathrm{obs}}^{95}}\right) .
$$

That is, $f$ is set to $1 / 15$, except if this choice would lead to an effective threshold below the minimum value of $0.01 \mathrm{~mm}$ for one of the fields. If $f=1 / 15$ leads to an effective threshold below $0.01 \mathrm{~mm}, f$ is chosen as the minimum value that keeps the effective threshold $\geq 0.01 \mathrm{~mm}$ for the forecast and the reference field.

\section{b. Intraensemble values of eSAL}

The individual members of an ensemble forecast are usually quite similar for short lead times, but what eSAL values are actually obtained if such rather similar fields are compared? In experiment 3 we verify the members $2-16$ 
of the CLEPS ensemble against the first CLEPS ensemble member for the smallest available lead times using eSAL; that is, the CLEPS members $2-16$ are treated as an ensemble forecast and member 1 as an observation. The aim of this experiment is to compare the magnitude of the eSAL components due to the forecast uncertainty expressed in the ensemble forecast to the magnitude of the eSAL components due to the analysis uncertainty tested in experiment 4.

\section{c. Intra-analysis comparison with eSAL}

The VERA ensemble is an experimental dataset; therefore, we want to know how similar it actually is to the deterministic VERA analysis in terms of eSAL. In experiment 4 we therefore treat the VERA ensemble as a model and verify it against the VERA deterministic analysis, again using eSAL. In addition, this experiment together with experiment 3 allows for extending the threshold sensitivity experiment in the sense that it shows how the eSAL components behave for fields that are supposed to be similar but are subject to perturbations reflecting forecast uncertainty or analysis uncertainty.

\section{d. Forecast verification}

Next, we conduct a series of forecast verification experiments using SAL distributions and eSAL.

For experiment 5 , we verify the hourly precipitation from the COSMO2 forecasts against each of the 50 VERA ensemble members. The forecast lead times range from 0 to $23 \mathrm{~h}$ depending on the time of the day. The VERA ensemble available for the three days of case 1 allows for a direct test of the behavior of SAL with respect to observation uncertainty within a forecast verification context.

In experiment 6, all 16 CLEPS members are first verified against the VERA analysis for 3-hourly precipitation forecasts, five lead-time ranges, and case 1 . Second, eSAL is used to verify the ensemble forecast against the VERA analysis. The eSAL results are compared to the median of the SAL component distributions and to score densities in $S-A, S-L$, and $A-L$ diagrams.

In experiment 7, the 3-hourly precipitation values of the COSMO2 and CLEPS models are verified against the deterministic VERA analysis and the ensemble VERA analysis using eSAL. This experiment reflects a setting that allows for comparison of the forecast performance of two models.

\section{Results}

\section{a. Threshold sensitivity experiments}

For each forecast-observation pair, SAL is calculated using several values of the threshold factor $f$. Figure 2 shows the differences in the threshold-dependent components $S$ and $L_{2}$ as a function of the difference in the threshold factor $f$ for all forecast-observation pairs. The median and interquartile ranges of the $S$ and $L_{2}$ differences are declining toward 0 for small threshold factor differences, so the average behavior seems stable. This result is different from Weniger and Friederichs (2016), who found unstable behavior in the average $S$ and $L_{2}$ component differences. We think that Weniger and Friederichs (2016) used actual thresholds below or equal to the minimum value of the field in some cases, which led to the unstable behavior they observed. The maximum differences for $S$ decline for small threshold factor differences but stay above 0.9 even for the smallest differences. The maximum differences of $L_{2}$ do not decline for small threshold factor differences, which means that situations showing the unstable behavior of $L_{2}$ do occur, but these cases are rare.

Figure 3 shows the results of the eSAL threshold sensitivity experiment. As in experiment 1 , the median $e S$ and $e L_{2}$ differences and the interquartile range of the $e S$ and $e L_{2}$ differences decline with small threshold factor differences and so does the maximum difference of $e S$ but not the maximum difference of $e L_{2}$. The smaller number of cases compared to experiment 1 leads to the peaks in the maximum $e L_{2}$ values. The peaks all correspond to the same verification date, 2100 UTC 21 June 2007. With more cases, the area between the spikes would probably be filled with other cases and give a picture similar to the deterministic case in Fig. 2. If experiment 1 is performed only with data from case 1 , the same kinds of peaks are observed (not shown). We conclude that the threshold sensitivity of eSAL is very similar to that of SAL and that the peaks in Fig. 3 are a symptom of a lack of data in the experiment and not a problem specific to eSAL.

\section{b. Intraensemble values of eSAL}

Figure 4 shows the SAL diagram of CLEPS ensemble members $2-16$ verified against the first CLEPS member using eSAL. The amplitude errors and the structure errors are distributed around zero, and the location errors are small. The spread in the $e A$ and $e S$ components is similar and on the order of \pm 0.65 . For the $e A$ component this spread corresponds roughly to double the domain-average precipitation amount in one field compared to the other.

\section{c. Intra-analysis comparison with eSAL}

Figure 5 shows SAL diagrams for verifying hourly and 3-hourly precipitation of the VERA ensemble against the VERA deterministic analysis during case 1 using eSAL (experiment 4). The hourly ensemble has a small 


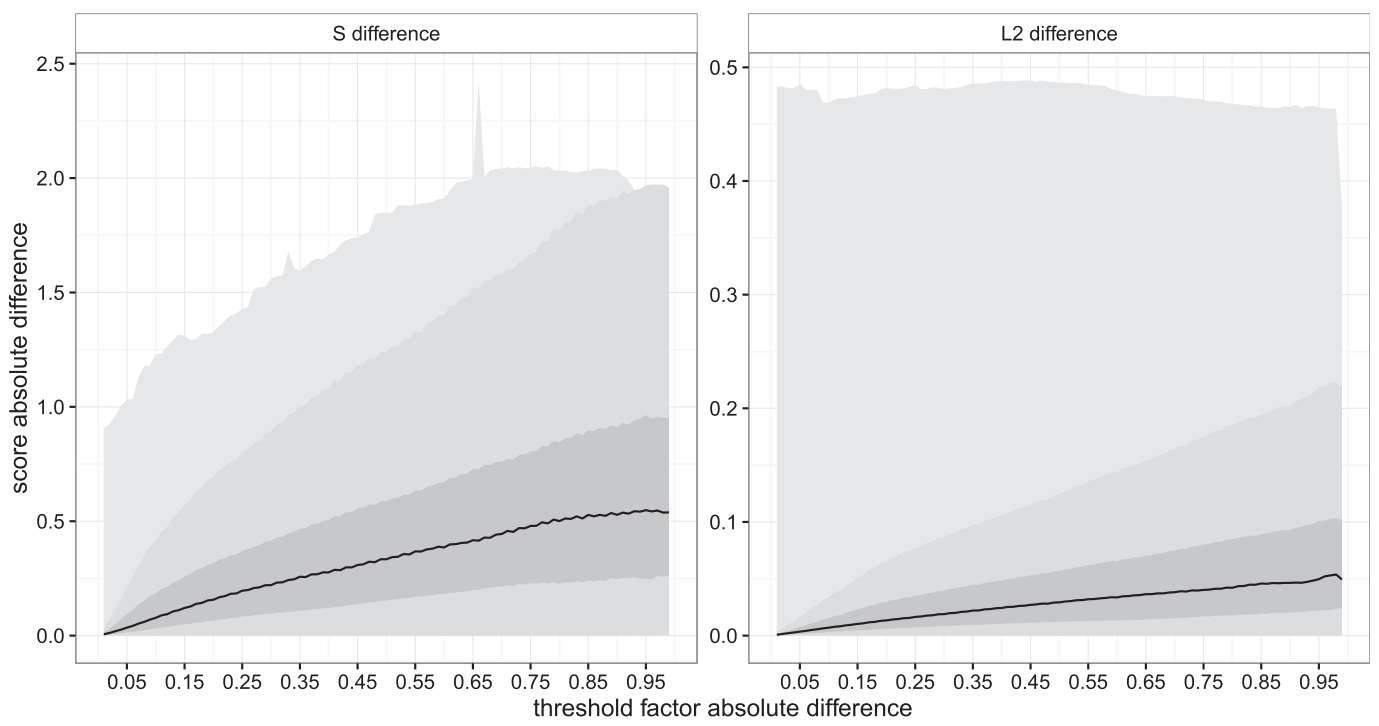

FIG. 2. Differences in the $S$ and $L_{2}$ components as a function of threshold factor differences $\Delta f$ verifying the hourly precipitation from COSMO2 against VERA for the 18 case study days. The median is shown in black, the interquartile range in dark gray, the range given by the minimum (maximum) of 1.5 times the interquartile range added to the third quartile (subtracted from the first quartile) and the maximum (minimum) in medium gray, and the full range in light gray.

positive amplitude bias with respect to the deterministic analysis and structure errors spreading widely for small domain-average precipitation amounts but clustering between 0 and +0.5 . That is, the hourly VERA ensemble tends to have slightly larger or a bit more flat features than the deterministic analysis, for cases with more domain-wide precipitation. For the 3-hourly ensemble there is nearly no amplitude bias and only one case with a very large structure error, while all other cases cluster around small positive values. The location errors are of the same order of magnitude as within the CLEPS ensemble (Fig. 4).

It has to be noted that the VERA ensemble is an experimental product rather than an operational product, and Gorgas and Dorninger (2012a) tested several ways of creating the perturbations of the precipitation

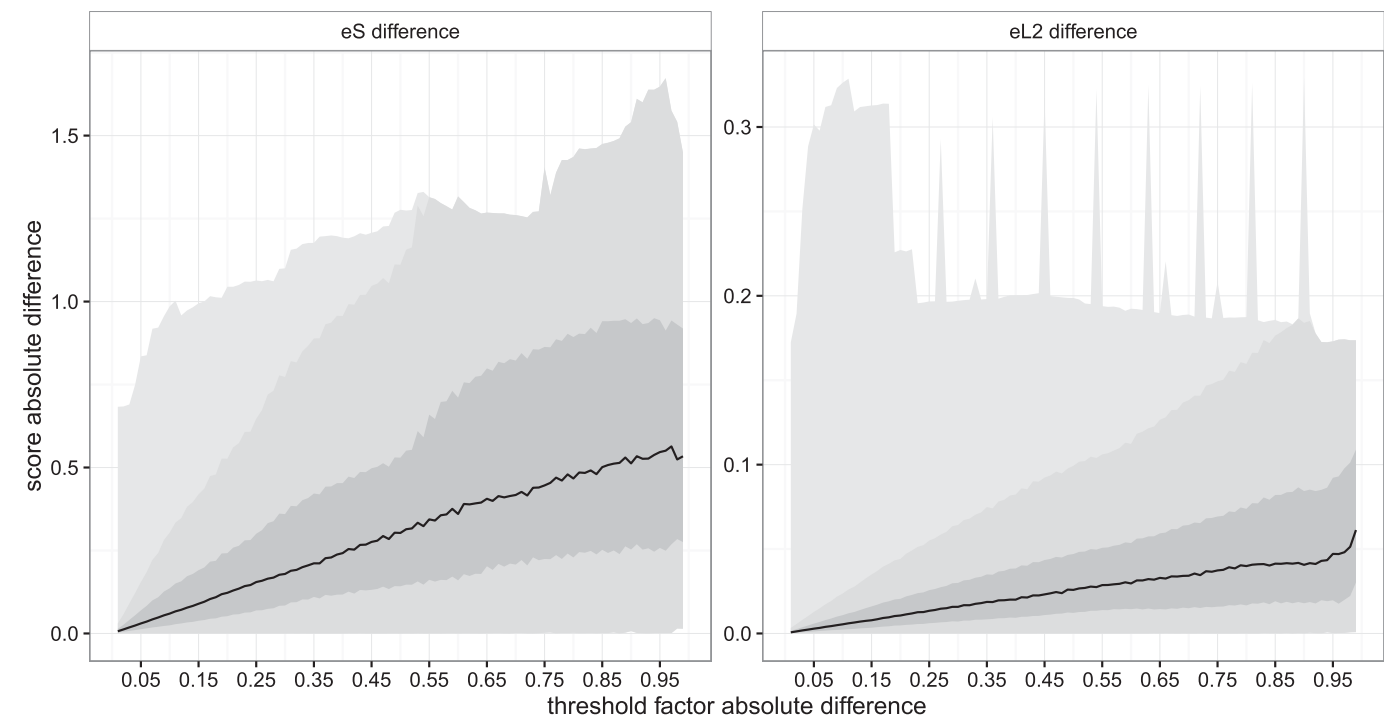

FIG. 3. As in Fig. 2, but for differences in the $e S$ and $e L_{2}$ components as a function of threshold factor differences $\Delta f$ for verifying the COSMO hourly forecasts for case 1 with the VERA ensemble analysis. 


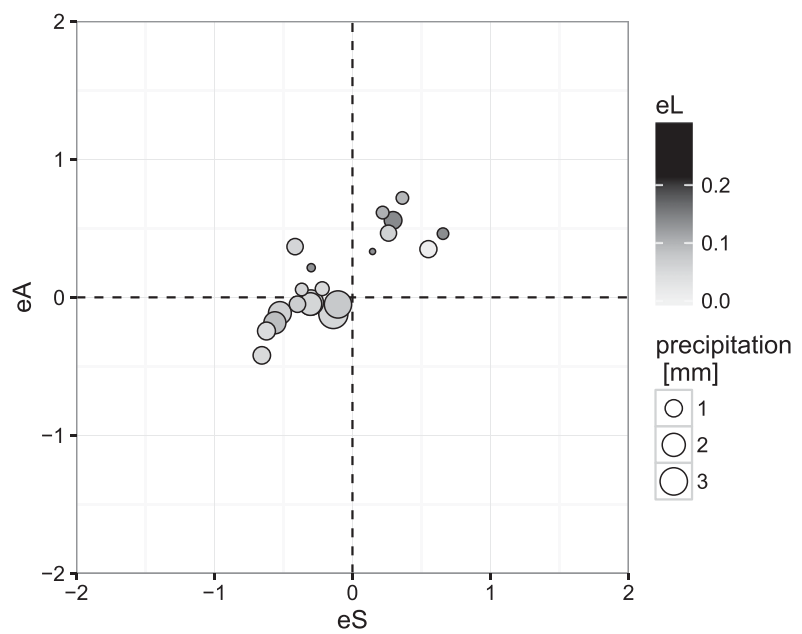

FIG. 4. SAL diagram for the verification of 3-hourly precipitation from CLEPS ensemble members 2-16 against CLEPS ensemble member 1 using eSAL. The size of the circles corresponds to the domain-average precipitation $(\mathrm{mm})$ in CLEPS member 1.

analysis. For example, another version of the VERA ensemble available within the MesoVICT project has a much larger amplitude bias and larger location and structure differences with the deterministic VERA analysis (not shown).

\section{d. Forecast verification}

In experiment 5 , the hourly COSMO2 forecasts are verified against each of the VERA ensemble members and the VERA ensemble as a whole. The lead time ranges from 0 to $23 \mathrm{~h}$ depending on the time of the day.
Figure 6 shows the resulting distributions of the SAL components for each hour of the three days studied, as well as the eSAL values.

Under the assumption that the VERA ensemble represents analysis uncertainty, the boxplots represent the uncertainty of the SAL components due to analysis uncertainty. The distributions of the $S$ and $L_{2}$ components are large for many time steps, with frequent outliers, especially for $L_{2}$, which means that analysis uncertainty leads to substantial uncertainty in the $S$ and $L_{2}$ components. Note that except for $L_{2}$ the variations of the SAL components with time are still dominant. Most of the time the eSAL values are close to the median of the SAL distribution for the $S$ and $A$ components. The $e L_{2}$ tends to be below the median of the $L_{2}$ distribution.

In experiment 6 , the 16 CLEPS ensemble members are verified individually against the VERA analysis. Figure 7 shows the distributions of the 16 resulting SAL values per time step and lead-time range for the verification of 3-hourly precipitation in five lead-time ranges.

As expected, the SAL component distributions are wider for long lead times than for short lead times for $A$ and $L$ but not so much for $S$. The ensemble members become more different in terms of precipitation quantities with longer lead times, which results in a larger spread in the $A$ component. Similarly, the locations of the fronts are more uncertain at longer lead times, which results in a larger spread of the $L$ component. The average scaled volumes of the $S$ component are partly a characteristic of the model itself that stays the same for longer lead times.

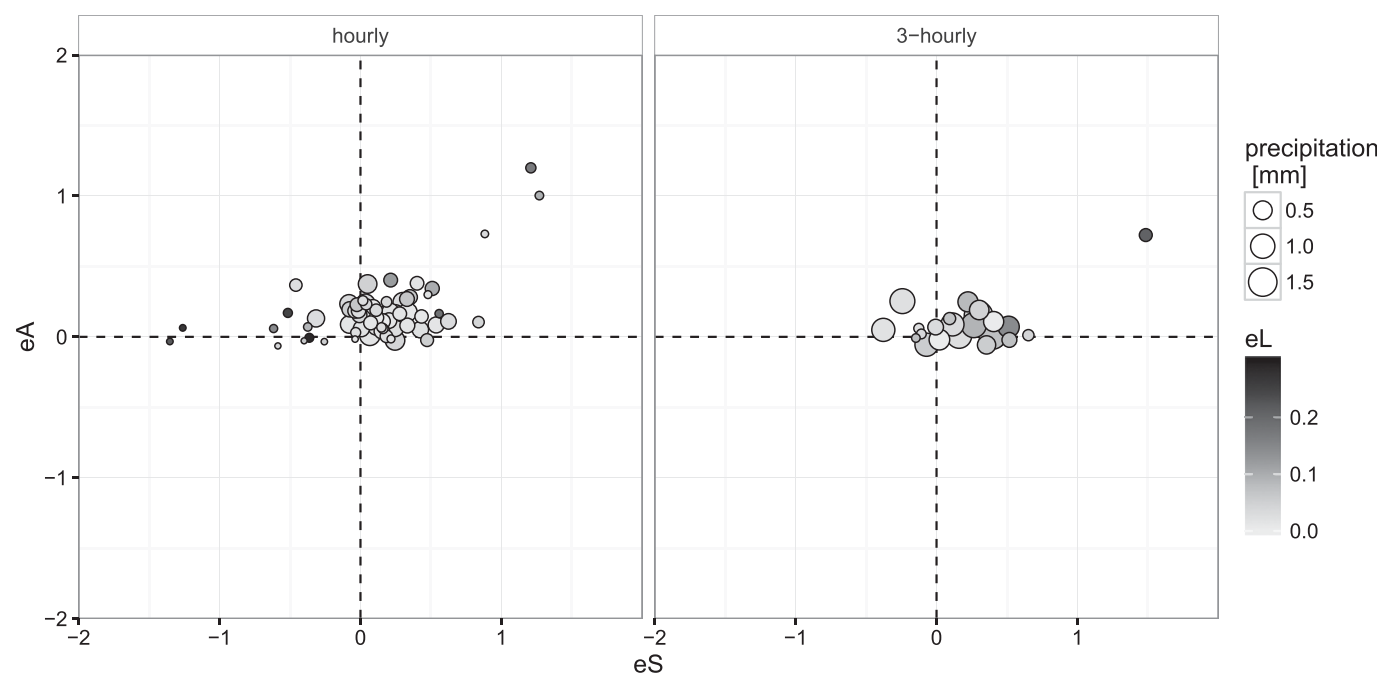

FIG. 5. SAL diagrams for the verification of (left) hourly and (right) 3-hourly precipitation from the VERA ensemble against the VERA deterministic analysis using eSAL. The sizes of the circles correspond to the domainaverage precipitation $(\mathrm{mm})$ in the deterministic VERA analysis. 


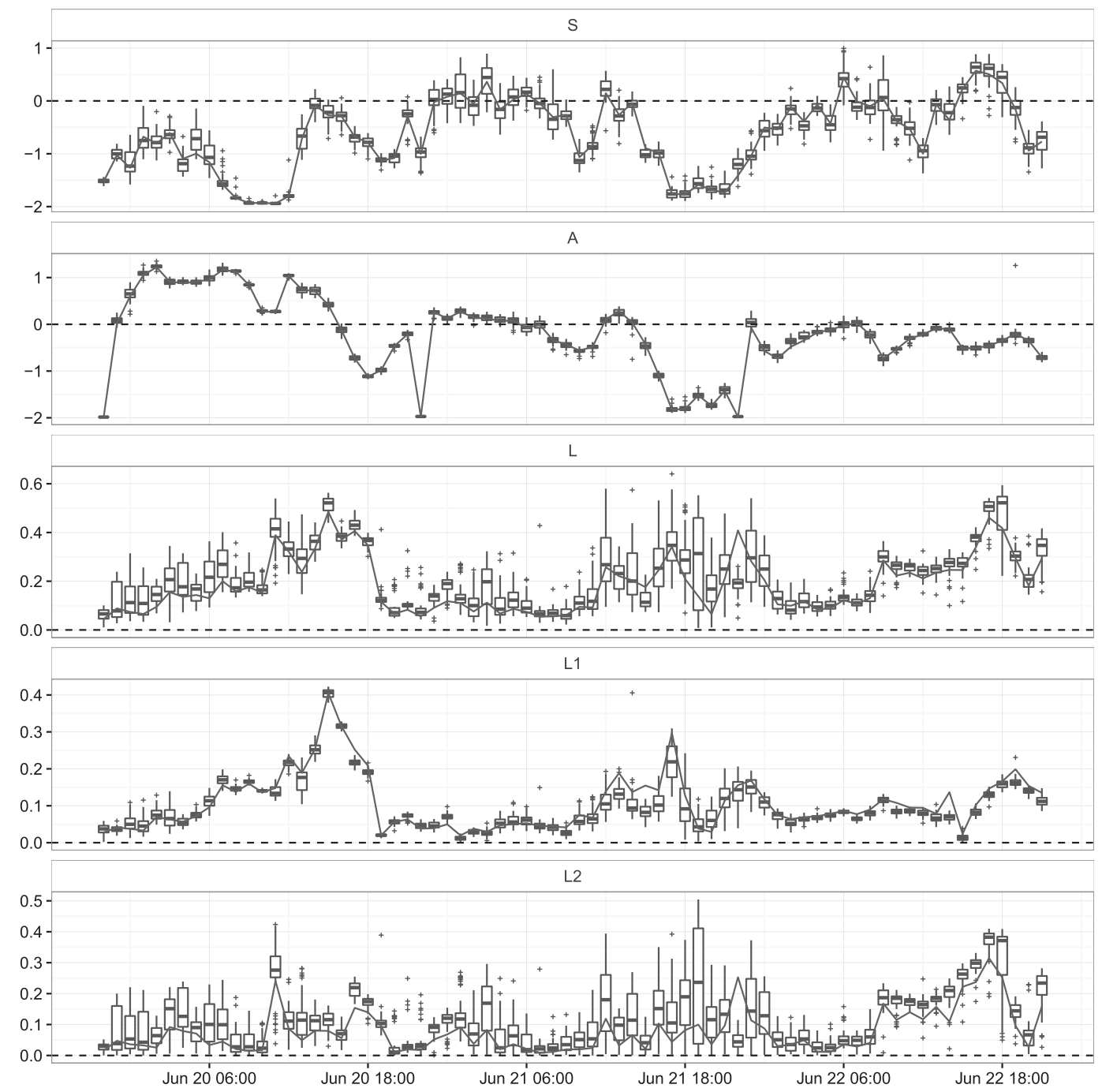

FIG. 6. Distributions of $S, A$, and $L$ for each hour during case 1, verifying the COSMO2 model against the VERA analysis ensemble (boxplots) and the corresponding eSAL values (solid lines).

Figure 7 also shows the eSAL components and the median of the standard SAL components for the five lead-time classes. The $e A$ and the $e S$ values tend to be close to the median of the standard SAL distributions. The $e L$, which does not take negative values, tends to be lower or equal to the median of the $L$ values. This behavior is related to the use of absolute distances and CRPS where the distance of the ensemble mean is smaller than or equal to the median of the distances most of the time except for situations when a far outlier does influence the mean and not the median. See, for example, $L_{1}$ at the 99-120-h lead-time range at the first time step. The $e L_{1}$ component is smaller than the median of $L_{1}$ when the spatial distribution of the center of the mass of the ensemble members is overlapping for the two ensembles and especially when the ensemble spread is rather large. In the presence of high uncertainty-here represented by the ensemble spread-the median of $L_{1}$ gets large even if the distributions of the centers of mass have a large overlapping area, which is not a desired effect, while $e L_{1}$ depends only on the ensemble mean center of mass and highlights the added information content of the ensemble forecast as compared to deterministic forecasts.

An issue with ensembles of deterministic SAL values is that even with only a few time steps, the number of points on the SAL diagram can become too high to give a meaningful picture if the ensemble forecasts or analysis ensembles used have a large number of ensemble members. One advantage of eSAL is that the number of points in the SAL diagrams does not depend on the ensemble size. However, we want to analyze the 


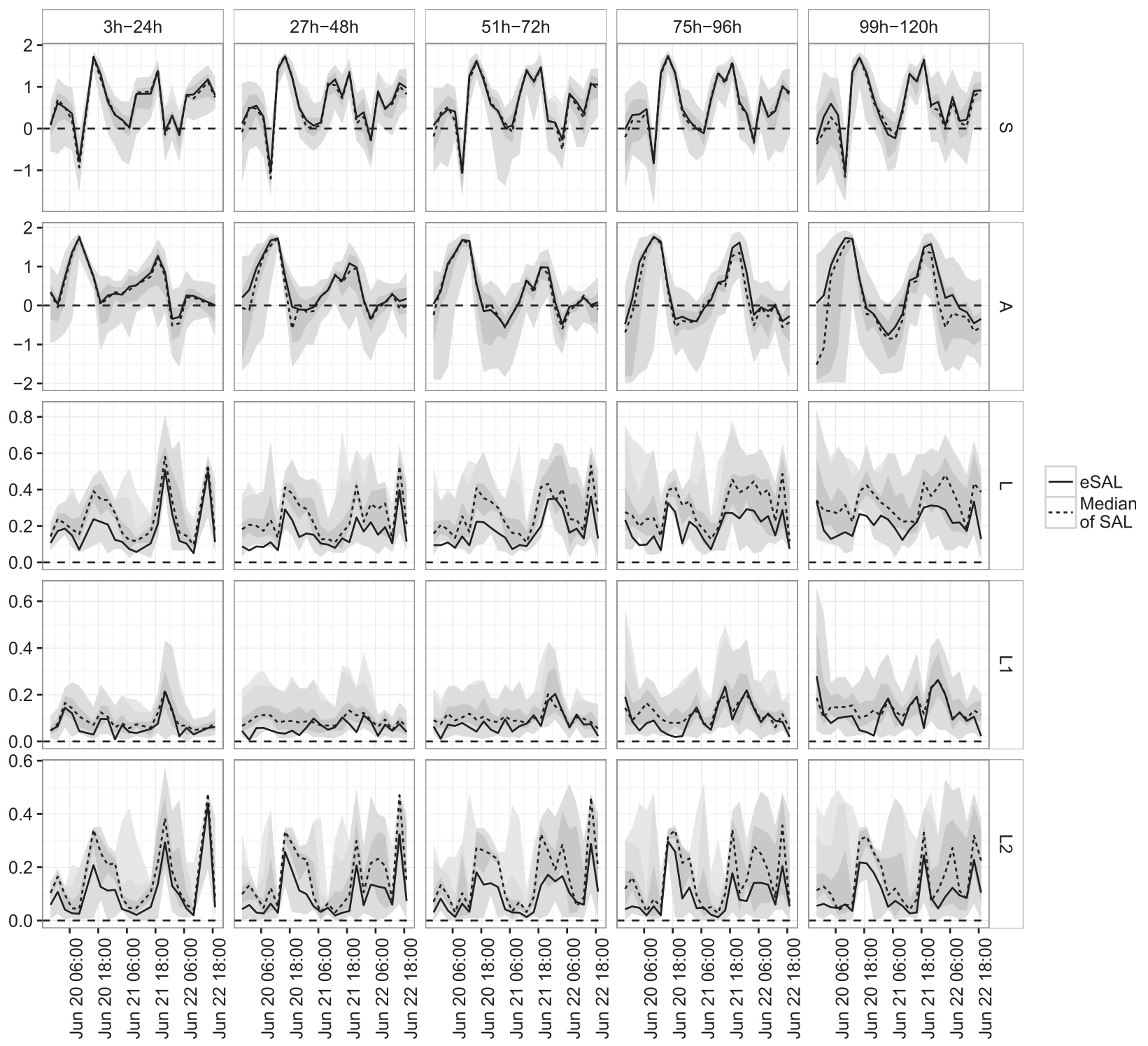

FIG. 7. SAL distribution and eSAL for verifying CLEPS 3-hourly forecasts with the VERA analysis. The eSAL corresponds to the solid line, and the dashed line is the median of the standard SAL. The light gray shading corresponds to the full range of standard SAL values and the darker shading to the interquartile range of the standard SAL.

distributions of deterministic SAL values as well. We therefore show density diagrams of pairs of two components in Fig. 8 for different lead-time classes (columns). The values for eSAL are added as red crosses for comparison. The density plots show that there is some overestimation of the feature size (positive $S$ values) for all lead-time classes. The total precipitation over the domain ( $A$ component) is slightly overestimated for the majority of the cases for low lead times. For higher lead times the whole range of $A$ values occurs. The $L$ component is overall rather small, especially for short lead times. However, it should be kept in mind that the $L$ values are relative to the domain size and that for large verification domains as used in this study even "small" $L$ values of, say, 0.1 already correspond to a displacement of roughly $100 \mathrm{~km}$. The eSAL values (red crosses in Fig. 8) reflect the distribution quite well at least for lead times not longer than 3 days, but because of the small number of cases (23) the concentration of points in certain areas of the diagram space is less obvious than for the density plots of standard SAL that are based on more points.

The results from the model intercomparison experiment 7 are shown in Fig. 9, which shows SAL diagrams 

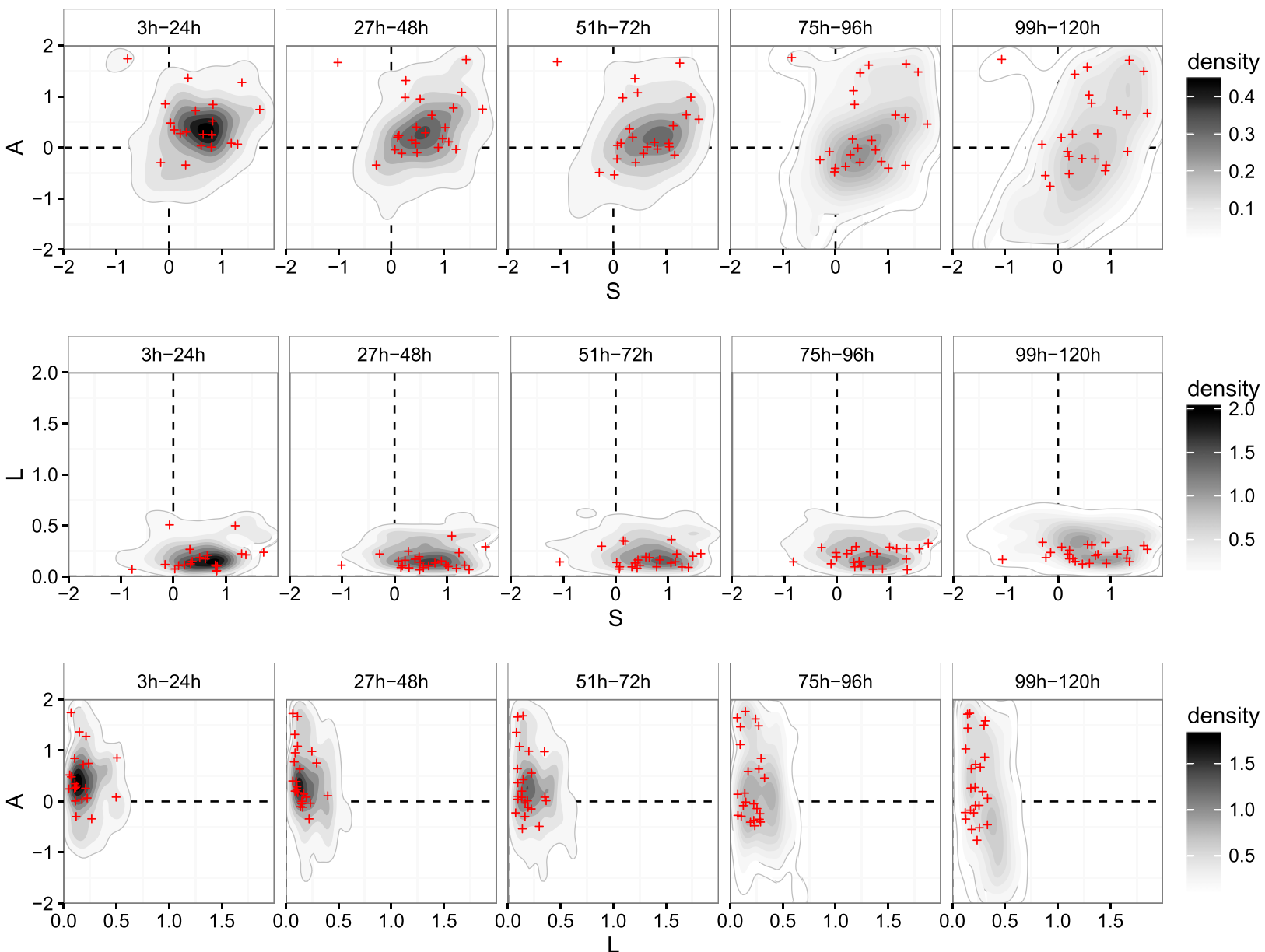

FIG. 8. Score density of SAL and the corresponding eSAL (red points) for CLEPS 3-hourly forecasts and VERA: (top) $S$ vs $A$, (middle) $S$ vs $L$, and (bottom) $L$ vs $A$.

for the verification of 3-hourly precipitation of the COSMO2 and CLEPS models against the deterministic VERA analysis and the VERA analysis ensemble using eSAL. The diagrams show that CLEPS tends to simulate higher domain average precipitation amounts than COSMO2 with larger or more flat structure, as expected for a model with lower resolution, and slightly lower location errors. With the VERA ensemble the location errors of the two models tend to be smaller. This result is coherent with Fig. 7 and shows that for many cases the forecasted location lies within the range of the analysis uncertainty. COSMO2 can be considered to be more accurate with respect to $e A$ than CLEPS. For cases with little domain-wide precipitation, COSMO2 tends to simulate too small features (strongly negative $e S$ ) while for the majority of cases the feature size corresponds rather well to the one in the analysis and the $e S$ values are distributed around zero. CLEPS tends to simulate too large or too flat features. CLEPS has slightly lower $e L$ values than COSMO2, which highlights the advantage of the ensemble forecast for predicting the location of precipitation.

\section{Discussion}

\section{a. Threshold sensitivity}

Wernli et al. (2008) have already pointed out that the choice of the threshold for the feature definition may have large impacts on the verification results under some circumstances, but they concluded based on tests varying the threshold factor between $f=1 / 13$ and $f=1 / 17$ that these cases should be rather rare. Recently, Weniger and Friederichs (2016) showed for spectral radiation data that small changes in the threshold are equivalent to taking observation uncertainty into account. They further showed that the threshold variations indeed severely impact the verification results and concluded that the 


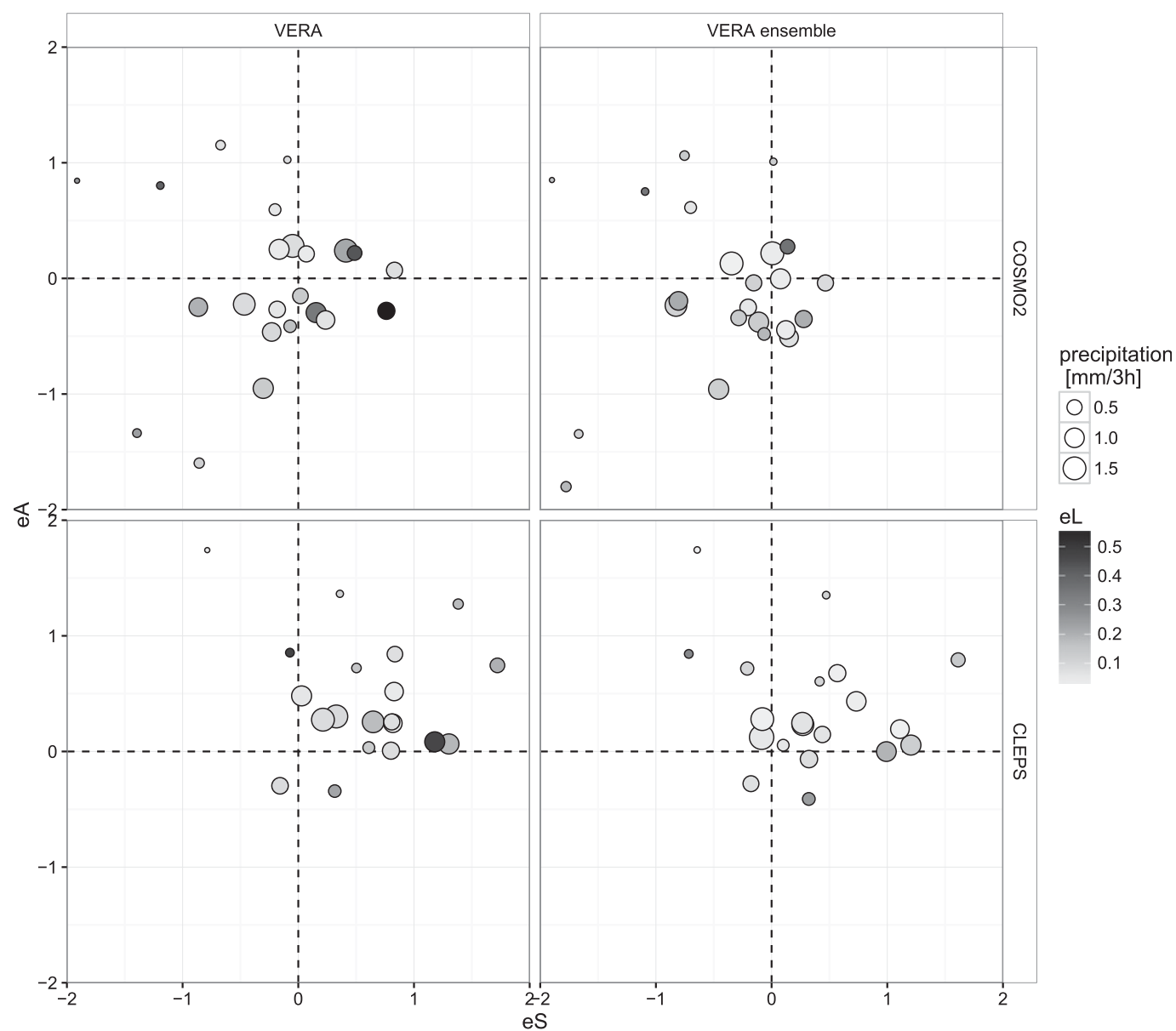

FIG. 9. The eSAL diagrams for the verification of 3-hourly precipitation from the (top) COSMO2 model and (bottom) CLEPS ensemble for the shortest available lead times against the (left) VERA deterministic analysis and (right) VERA ensemble.

threshold-dependent $S$ and $L_{2}$ components are not well suited for the verification of ensembles as a collection of deterministic forecasts. We have reasons to think that some of the results of Weniger and Friederichs (2016) are due to cases where the effective threshold, that is, $R^{95} \times f$, is equal or below the minimum value of the data. One can imagine that the feature characteristics of $S$ and $L_{2}$ are constant as long as the effective threshold is below or equal to the minimum value of the field and may change substantially between the case where the whole domain is considered as one feature and the case where the effective threshold is slightly above the minimum value, and therefore several features can be in place. We argue that those cases are quite easy to avoid even in an automatic setting and that effective thresholds equal or below the minimum value of the field are not meaningful for the SAL verification anyway and should therefore be avoided.

A similar issue occurs when the resolution of the data is limited. For example, the precipitation data used here are given with two significant digits, that is, at a numerical resolution of $0.01 \mathrm{~mm}$. For cases where $R^{95}$ is small, very small differences in $f$ may lead to effective threshold differences smaller than $0.01 \mathrm{~mm}$. This rounding error leads to artificial differences of zero and artificially high $S$ and $L_{2}$ differences when a 0.01 step is passed.

Arbogast et al. (2016) proposed an alternative to the commonly used object definition with thresholds based on an object detection algorithm using a set of reference histograms. Each type of precipitating object (e.g., front, mesoscale convective system) has a typical precipitation intensity distribution, its reference histogram. Arbogast et al.'s algorithm searches for contours that encompass regions where the precipitation intensity distribution matches one of the reference distributions. Such an approach may lead to more meaningful features, but includes higher computational costs.

\section{b. Observation uncertainty}

The results from the threshold sensitivity experiment (Fig. 2), the intraensemble and intra-analysis experiments 
(Figs. 4 and 5), and the SAL component distributions due to analysis uncertainty (Fig. 6) show that SAL and eSAL are highly sensitive to observation uncertainty. There are two main reasons for the high sensitivity of SAL and eSAL to observation uncertainty. The first one is related to the definition of the objects as explained by Weniger and Friederichs (2016); that is, observation uncertainty can lead to different numbers of objects (e.g., flat objects that are present or not, fusion of objects that severely changes the average size and shape characteristics). The second reason is related to the fact that the SAL/eSAL components are relative measures. The consequence is that for cases with little precipitation over the domain small absolute changes can lead to large relative changes in the SAL components. Our enhanced SAL diagrams, where the point size depends on the mean precipitation amount, help to visualize this second point. For example for the verification of the COSMO2 forecasts in Fig. 9 the cases with high precipitation amounts tend to cluster in a quite limited area of the diagram, while cases with small precipitation amounts are more spread out across the diagram. Especially when only a few cases are visualized, the enhanced SAL diagram helps to focus on the most important precipitation events. The enhanced SAL diagrams might be less useful when a much larger number of cases are visualized and a higher concentration of points in a specific area of the diagram becomes more evident anyway, and the overlapping of the larger points becomes more of an issue.

Gorgas and Dorninger (2012b) looked at the traditional verification statistics correlation and bias-corrected RMSE over the three days of case 1 . They compared their variations over time with their variations due to perturbations of the analysis. They found that the variations due to the perturbations of the analysis are in the order of $20 \%-40 \%$ of the temporal variation. Comparing the size of the boxplots in Fig. 6 with their temporal evolution and the dispersion of the points in the SAL diagram in Fig. 9 with the dispersion in Fig. 5 suggests that the variation of the eSAL components due to the perturbed reference data is on the order of $20 \%-50 \%$ of their temporal variations, where the highest relative variations can be observed for $e L$ and the lowest relative variations for $e A$. The variations of $e L$ and $e S$ are thus slightly higher than the variations of the correlation and bias-corrected RMSE found by Gorgas and Dorninger (2012b). For $e A$, the variations due to analysis perturbations are very similar to the ones of the traditional scores.

\section{c. Domain size}

The SAL values strongly depend on the size of the verification domain. This dependence is most evident for the $L$ component that is defined relative to the largest distance between two domain borders. Unlike methods that match features, $L$ is not a measure for the displacement of a specific structure. The domain has to be large enough to find at least one feature in both the forecast and the analysis field. On the other hand, a too large verification domain makes the interpretation of the SAL components more difficult, since features belonging to distinct meteorological systems are more likely to be present.

\section{d. Verification of probability fields}

A part of the ensemble performance evaluation is the verification of probabilities. It is tempting to apply SAL to fields of exceedance probabilities similar to the ones used by Schwartz et al. (2017). Unfortunately, looking at probabilities with SAL is not as straightforward as it seems at first sight. Specifically, simple exceedance probabilities at each grid cell render the $S$ component completely meaningless. For example, one cannot distinguish between cases where one ensemble member has a large area exceeding the precipitation threshold or cases where many members each have a small area exceeding the precipitation threshold but at neighboring grid cells, which in total leads to the same spatial field of probability with completely different precipitation structures in the ensemble. This issue might be solved by using a neighborhood approach for the definition of the exceedance probabilities, but, then again, $S$ accounts only for structures of the size of the neighborhood. Concerning the $A$ component there are issues with the interpretation. Specifically, how to interpret a relative difference in the domain-wide probability mass? The exceedance probability object approach works well for location-related indices like $L_{1}$ or the ones Schwartz et al. (2017) looked at, but designing meaningful structure indices based on probabilities is far less obvious. We therefore do not recommend applying SAL to simple exceedance probability fields.

\section{Conclusions}

This paper proposes an ensemble extension of the structure-amplitude-location method (eSAL) for the spatial verification of ensemble precipitation fields. The advantages of the ensemble version include 1) the number of scores for an ensemble forecast is equal to the number for a deterministic one, and therefore the comparison becomes easier; in addition, eSAL is equal to SAL in the deterministic case, and 2) the computation time is much shorter than calculating SAL values for all combinations of forecast ensemble 
members and analysis ensemble members. On the downside, the information on the variability of the ensemble is lost. Our enhanced SAL diagrams with the point size being proportional to the mean nonzero precipitation over the reference field allow for distinguishing cases with little precipitation from cases with high precipitation amounts in the SAL diagram and therefore help to diagnose potentially different behavior for different intensities.

The lessons learned from the threshold sensitivity tests are that any automated procedure to determine thresholds should avoid meaningless thresholds or threshold intervals, for example, thresholds below the minimum value of the field in question or intervals smaller than the resolution of the data. Using independent thresholds for the forecast and the analysis field is important to rendering the $S$ and $L_{2}$ components less dependent on overall intensity errors that are already captured by the $A$ component.

Experiment 7 highlights the flexibility of eSAL: eSAL can be used to verify ensemble forecasts against a deterministic analysis, to compare deterministic forecasts and ensemble forecasts of different sizes, and can even use an analysis ensemble for the verification of deterministic forecasts or ensemble forecasts if such an analysis ensemble is available.

Acknowledgments. The presented work was part of a Ph.D. project (Radanovics 2014) cofunded by Irstea and the Compagnie Nationale du Rhône (CNR). This work was further supported by the EU FP7 COMPLEX (Grant Agreement 308601) and an ERC A2C2 grant (Grant 338965). We thank Manfred Dorninger for the fruitful discussions on the VERA ensemble; the editor, Zhaoxia $\mathrm{Pu}$, for the very efficient handling of the manuscript during the review process; and three anonymous referees for their constructive comments that helped to improve the paper. Analysis and graphics were done using the R software environment (R Core Team 2016) with the packages SpatialVx (Gilleland 2016), ggplot2 (Wickham 2009), RColorBrewer (Neuwirth 2014), doMC (Calaway et al. 2015a), foreach (Calaway et al. 2015b), reshape2 (Wickham 2007), plyr (Wickham 2011), and rworldmap (South 2011).

\section{APPENDIX}

\section{Schemata of the $e L$ Component Calculation}

For the $e L_{1}$ calculation the $x$ coordinates and the $y$ coordinates of the center of mass of the ensemble members (Fig. A1, red dots) are averaged, resulting in

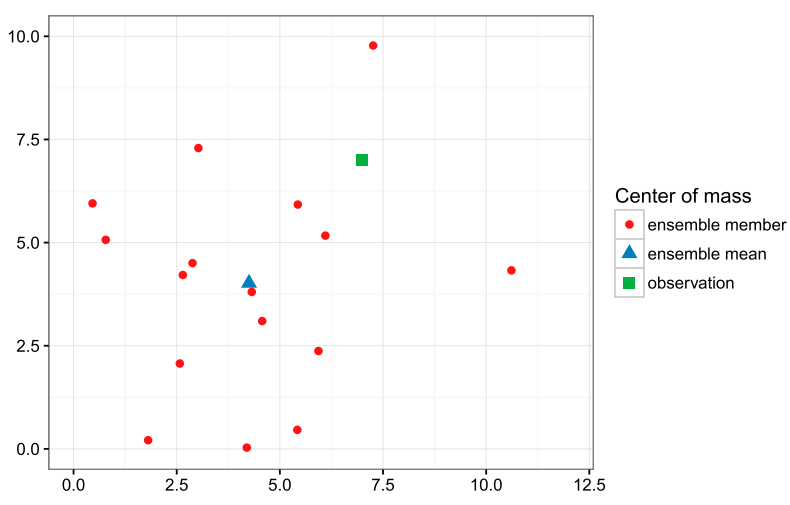

FIG. A1. The distance between the blue triangle and the green square divided by 12.5 (domain size) is $e L_{1}$. The blue triangle is the average of the red dots.

the blue triangle in Fig. A1. The difference between this ensemble average center of mass and the center of mass of the observations (the green square in Fig. A1) divided by the domain size is $e L_{1}$. Figures $\mathrm{A} 2$ and $\mathrm{A} 3$ illustrate the calculation of $e L_{2}$ in two steps. First, for each ensemble member (colors in Figs. A2 and A3) the mean distance between the individual feature centers (small symbols) to the total center of mass of the member in question (large symbols) is calculated. Note that the large dots in Fig. A2 correspond conceptually to the red dots in Fig. A1. The large black square of the observation corresponds to the green square in Fig. A1. Second, $e L_{2}$ is calculated as the CRPS between the two empirical cumulative distribution functions of the mean feature distances shown in Fig. A3, with all the values divided by the domain length.

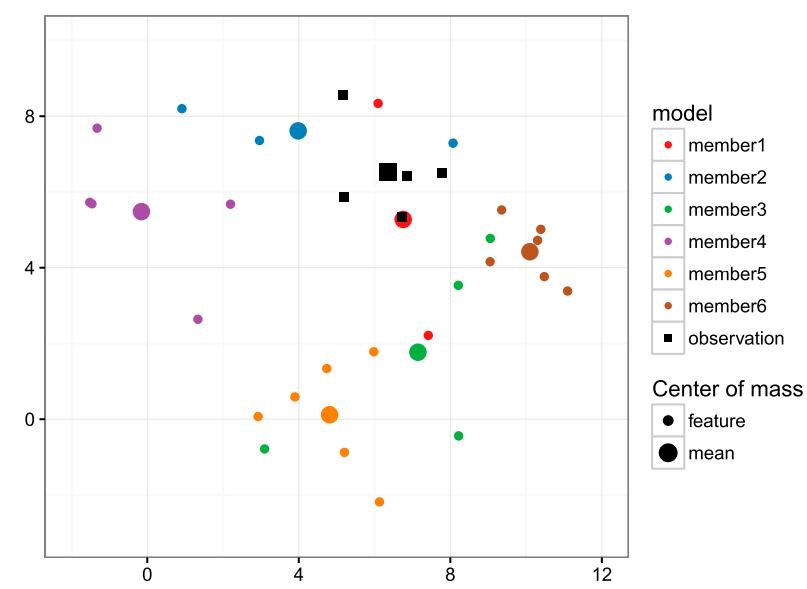

FIG. A2. For $e L_{2}$ we calculate first for each member (colors) the mean distance between the individual feature centers (small symbols) and the total center of mass of the member in question (large symbols). 


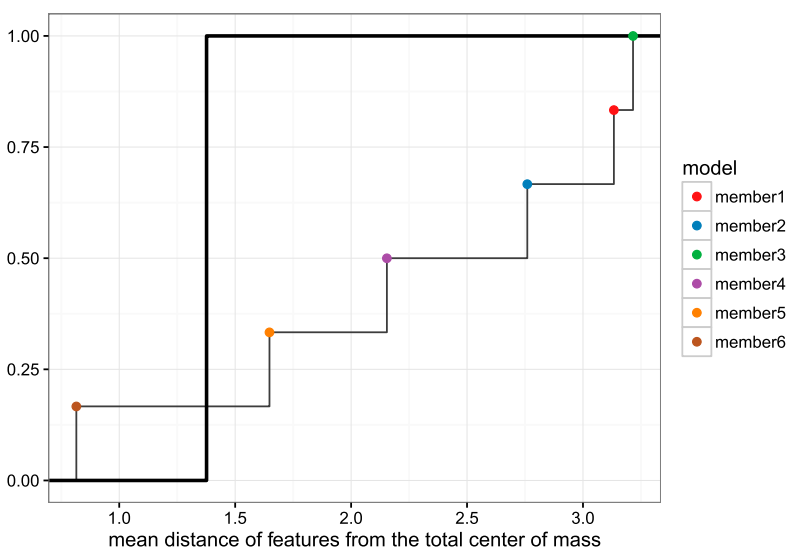

FIG. A3. Empirical cumulative distribution of the mean feature distance over all members and the observed mean distance, as shown by the thick black line.

\section{REFERENCES}

AghaKouchak, A., A. Behrangi, S. Sorooshian, K. Hsu, and E. Amitai, 2011: Evaluation of satellite-retrieved extreme precipitation rates across the central United States. J. Geophys. Res., 116, D02115, https://doi.org/10.1029/2010JD014741.

Ahijevych, D., E. Gilleland, B. G. Brown, and E. E. Ebert, 2009: Application of spatial verification methods to idealized and NWP-gridded precipitation forecasts. Wea. Forecasting, 24, 1485-1497, https://doi.org/10.1175/2009WAF2222298.1.

Ament, F., and M. Arpagaus, 2009: Metadata for 'dphase_cosmoch2: COSMO model forecasts $(2.2 \mathrm{~km})$ run by MeteoSwiss for the MAP D-PHASE project.' World Data Center for Climate, accessed 21 January 2014, https://doi.org/10.1594/ WDCC/dphase_cosmoch2.

Arbogast, P., O. Pannekoucke, L. Raynaud, R. Lalanne, and E. Mémin, 2016: Object-oriented processing of CRM precipitation forecasts by stochastic filtering. Quart. J. Roy. Meteor. Soc., 142, 2827-2838, https://doi.org/10.1002/qj.2871.

Aspelien, T., T. Iversen, J. B. Bremnes, and I.-L. Frogner, 2011: Short-range probabilistic forecasts from the Norwegian limitedarea EPS: Long-term validation and a polar low study. Tellus, 63A, 564-584, https://doi.org/10.1111/j.1600-0870.2010.00502.x.

Baldauf, M., A. Seifert, J. Förstner, D. Majewski, M. Raschendorfer, and T. Reinhardt, 2011: Operational convective-scale numerical weather prediction with the COSMO model: Description and sensitivities. Mon. Wea. Rev., 139, 3887-3905, https://doi.org/ 10.1175/MWR-D-10-05013.1.

Barrett, A. I., S. L. Gray, D. J. Kirshbaum, N. M. Roberts, D. M. Schultz, and J. G. Fairman, 2015: Synoptic versus orographic control on stationary convective banding. Quart. J. Roy. Meteor. Soc., 141, 1101-1113, https://doi.org/10.1002/qj.2409.

Ben Bouallègue, Z., and S. E. Theis, 2014: Spatial techniques applied to precipitation ensemble forecasts: From verification results to probabilistic products. Meteor. Appl., 21, 922-929, https://doi.org/10.1002/met.1435.

Bica, B., and Coauthors, 2007: Thermally and dynamically induced pressure features over complex terrain from high-resolution analyses. J. Appl. Meteor. Climatol., 46, 50-65, https://doi.org/ 10.1175/JAM2418.1.
Bowler, N. E., 2006: Explicitly accounting for observation error in categorical verification of forecasts. Mon. Wea. Rev., 134, 1600-1606, https://doi.org/10.1175/MWR3138.1.

_ 2007: Numerical weather prediction accounting for the effect of observation errors on verification of MOGREPS. NWP Tech. Rep. 506, Met Office, 7 pp., http://research.metoffice. gov.uk/research/nwp/publications/papers/technical_reports/ reports/506.pdf.

Briggs, W. M., and R. A. Levine, 1997: Wavelets and field forecast verification. Mon. Wea. Rev., 125, 1329-1341, https://doi.org/ 10.1175/1520-0493(1997)125<1329:WAFFV > 2.0.CO;2.

Brown, T. A., 1974: Admissible scoring systems for continuous distributions. Rand Publ. P-5235, Rand Paper Series, The Rand Corporation, Santa Monica, CA, https://www.rand.org/ pubs/papers/P5235.html.

Calaway, R., Revolution Analytics, and S. Weston, 2015a: DoMC: Foreach parallel adaptor for "parallel," version 1.3.4. R package, https://CRAN.R-project.org/package $=$ doMC.

$\longrightarrow, \ldots$, and -2015 b: Foreach: Provides Foreach looping construct for R, version 1.4.3. R package, https://CRAN.R-project.org/ package $=$ foreach.

Candille, G., and O. Talagrand, 2008: Impact of observational error on the validation of ensemble prediction systems. Quart. J. Roy. Meteor. Soc., 134, 959-971, https://doi.org/10.1002/qj.268.

Casati, B., G. Ross, and D. B. Stephenson, 2004: A new intensityscale approach for the verification of spatial precipitation forecasts. Meteor. Appl., 11, 141-154, https://doi.org/10.1017/ S1350482704001239.

Clark, A. J., R. G. Bullock, T. L. Jensen, M. Xue, and F. Kong, 2014: Application of object-based time-domain diagnostics for tracking precipitation systems in convection-allowing models. Wea. Forecasting, 29, 517-542, https://doi.org/ 10.1175/WAF-D-13-00098.1.

Davis, C., B. Brown, and R. Bullock, 2006: Object-based verification of precipitation forecasts. Part I: Methodology and application to mesoscale rain areas. Mon. Wea. Rev., 134, 1772-1784, https:// doi.org/10.1175/MWR3145.1.

, — _ _ , and J. Halley-Gotway, 2009: The Method for Object-Based Diagnostic Evaluation (MODE) applied to numerical forecasts from the 2005 NSSL/SPC Spring Program. Wea. Forecasting, 24, 1252-1267, https://doi.org/10.1175/ 2009WAF2222241.1.

Demargne, J., J. Brown, Y. Liu, D.-J. Seo, L. Wu, Z. Toth, and Y. Zhu, 2010: Diagnostic verification of hydrometeorological and hydrologic ensembles. Atmos. Sci. Lett., 11, 114-122, https://doi.org/10.1002/asl.261.

Denis, B., J. Coté, and R. Laprise, 2002: Spectral decomposition of two-dimensional atmospheric fields on limited-area domains using the discrete cosine transform (DCT). Mon. Wea. Rev., 130, 1812-1829, https://doi.org/10.1175/1520-0493(2002)130<1812: SDOTDA $>2.0 . \mathrm{CO} ; 2$.

Dey, S. R. A., G. Leoncini, N. M. Roberts, R. S. Plant, and S. Migliorini, 2014: A spatial view of ensemble spread in convection permitting ensembles. Mon. Wea. Rev., 142, 40914107, https://doi.org/10.1175/MWR-D-14-00172.1.

, R. S. Plant, N. M. Roberts, and S. Migliorini, 2016: Assessing spatial precipitation uncertainties in a convective-scale ensemble. Quart. J. Roy. Meteor. Soc., 142, 2935-2948, https:// doi.org/10.1002/qj.2893.

Dorninger, M., and T. Gorgas, 2013: Comparison of NWP-model chains by using novel verification methods. Meteor. Z., 22, 373-393, https://doi.org/10.1127/0941-2948/2013/0488. 
- M. Mittermaier, E. Gilleland, E. Ebert, B. Brown, and L. Wilson, 2013: MesoVICT: Mesoscale Verification Inter-Comparison over Complex Terrain. NCAR Tech. Note NCAR/TN-505+STR, 23 pp., https://doi.org/10.5065/D6416V21 .

Duc, L., K. Saito, and H. Seko, 2013: Spatial-temporal fractions verification for high-resolution ensemble forecasts. Tellus, 65A, 18171, https://doi.org/10.3402/tellusa.v65i0.18171.

Ebert, E. E., 2008: Fuzzy verification of high-resolution gridded forecasts: A review and proposed framework. Meteor. Appl., 15, 51-64, https://doi.org/10.1002/met.25.

_- and J. McBride, 2000: Verification of precipitation in weather systems: Determination of systematic errors. J. Hydrol., 239, 179-202, https://doi.org/10.1016/S0022-1694(00)00343-7.

— ification. Meteor. Appl., 20, 130-139, https://doi.org/10.1002/ met.1392.

Farchi, A., M. Bocquet, Y. Roustan, A. Mathieu, and A. Quérel, 2016: Using the Wasserstein distance to compare fields of pollutants: Application to the radionuclide atmospheric dispersion of the Fukushima-Daiichi accident. Tellus, 68B, 31682, https://doi.org/10.3402/tellusb.v68.31682.

Fox, N. I., A. C. Micheas, and Y. Peng, 2016: Applications of Bayesian Procrustes shape analysis to ensemble radar reflectivity nowcast verification. Atmos. Res., 176-177, 75-86, https://doi.org/10.1016/j.atmosres.2016.02.001.

Gallus, W. A., Jr., 2010: Application of object-based verification techniques to ensemble precipitation forecasts. Wea. Forecasting, 25, 144-158, https://doi.org/10.1175/2009WAF2222274.1.

Geiß, S., 2015: Comparison of spatial verification methods. B.S. thesis, Ludwig-Maximilians-University, Munich Meteorological Institute, Munich, Germany, 43 pp., http://www.ral. ucar.edu/projects/icp/ba2015_geiss_stefan.pdf.

Germann, U., M. Berenguer, D. Sempere-Torres, and M. Zappa, 2009: REAL_Ensemble radar precipitation estimation for hydrology in a mountainous region. Quart. J. Roy. Meteor. Soc., 135, 445-456, https://doi.org/10.1002/qj.375.

Gilleland, E., 2011: Spatial forecast verification: Baddeley's delta metric applied to the ICP test cases. Wea. Forecasting, 26, 409415, https://doi.org/10.1175/WAF-D-10-05061.1.

_ 2015: Verification: Weather forecast verification utilities, version 1.42. R package, Research Applications Laboratory, NCAR, Boulder, CO, https://CRAN.R-project.org/package= verification. , 2016: SpatialVx: Spatial forecast verification, version 0.5. R package, http://CRAN.R-project.org/package $=$ SpatialVx.

_- 2017: A new characterization within the spatial verification framework for false alarms, misses, and overall patterns. Wea. Forecasting, 32, 187-198, https://doi.org/10.1175/ WAF-D-16-0134.1.

—-, D. Ahijevych, B. G. Brown, B. Casati, and E. E. Ebert, 2009: Intercomparison of spatial forecast verification methods. Wea. Forecasting, 24, 1416-1430, https://doi.org/ 10.1175/2009WAF2222269.1.

— warping. NCAR Tech. Note NCAR/TN-482+STR, 23 pp., https://opensky.ucar.edu/islandora/object/technotes \%3A495.

Gneiting, T., and A. E. Raftery, 2007: Strictly proper scoring rules, prediction, and estimation. J. Amer. Stat. Assoc., 102, 359-378, https://doi.org/10.1198/016214506000001437.

Goessling, H. F., and T. Jung, 2018: A probabilistic verification score for contours: Methodology and application to arctic iceedge forecasts. Quart. J. Roy. Meteor. Soc., https://doi.org/ 10.1002/qj.3242, in press.
Gofa, F., D. Boucouvala, P. Louka, and H. Flocas, 2017: Spatial verification approaches as a tool to evaluate the performance of high resolution precipitation forecasts. Atmos. Res., 208, 78-87, https://doi.org/10.1016/j.atmosres.2017.09.021.

Good, I. J., 1952: Rational decisions. J. Roy. Stat. Soc., 14B, 107114.

Gorgas, T., and M. Dorninger, 2012a: Concepts for a patternoriented analysis ensemble based on observational uncertainties. Quart. J. Roy. Meteor. Soc., 138, 769-784, https:// doi.org/10.1002/qj.949.

$\longrightarrow$, and -2012 b: Quantifying verification uncertainty by reference data variation. Meteor. Z., 21, 259-277, https://doi.org/ 10.1127/0941-2948/2012/0325.

Griffin, S. M., J. A. Otkin, C. M. Rozoff, J. M. Sieglaff, L. M. Cronce, and C. R. Alexander, 2017: Methods for comparing simulated and observed satellite infrared brightness temperatures and what do they tell us? Wea. Forecasting, 32, 5-25, https://doi.org/10.1175/WAF-D-16-0098.1.

Hagedorn, R., T. M. Hamill, and J. S. Whitaker, 2008: Probabilistic forecast calibration using ECMWF and GFS ensemble reforecasts. Part I: Two-meter temperatures. Mon. Wea. Rev., 136, 2608-2619, https://doi.org/10.1175/2007MWR2410.1.

Haiden, T., A. Kann, C. Wittmann, G. Pistotnik, B. Bica, and C. Gruber, 2011: The Integrated Nowcasting through Comprehensive Analysis (INCA) system and its validation over the eastern Alpine region. Wea. Forecasting, 26, 166-183, https:// doi.org/10.1175/2010WAF2222451.1.

Hanley, K. E., D. J. Kirshbaum, N. M. Roberts, and G. Leoncini, 2013: Sensitivities of a squall line over central Europe in a convective-scale ensemble. Mon. Wea. Rev., 141, 112-133, https://doi.org/10.1175/MWR-D-12-00013.1.

Hardy, J., J. J. Gourley, P.-E. Kirstetter, Y. Hong, F. Kong, and Z. L. Flamig, 2016: A method for probabilistic flash flood forecasting. J. Hydrol., 541A, 480-494, https://doi.org/10.1016/ j.jhydrol.2016.04.007.

Hersbach, H., 2000: Decomposition of the continuous ranked probability score for ensemble prediction systems. Wea. Forecasting, 15, 559-570, https://doi.org/10.1175/1520-0434(2000)015<0559: DOTCRP $>2.0 . \mathrm{CO} ; 2$.

Jordan, A., F. Krueger, and S. Lerch, 2017: ScoringRules: Scoring rules for parametric and simulated distribution forecasts, version 0.9.2. $\mathrm{R}$ package, https://CRAN.R-project.org/package $=$ scoringRules.

Keil, C., and G. C. Craig, 2007: A displacement-based error measure applied in a regional ensemble forecasting system. Mon. Wea. Rev., 135, 3248-3259, https://doi.org/10.1175/MWR3457.1.

— ploying an optical flow technique. Wea. Forecasting, 24, 12971308, https://doi.org/10.1175/2009WAF2222247.1.

Lange, H., and G. C. Craig, 2014: The impact of data assimilation length scales on analysis and prediction of convective storms. Mon. Wea. Rev., 142, 3781-3808, https://doi.org/10.1175/ MWR-D-13-00304.1.

Marsigli, C., F. Boccanera, A. Montani, and T. Paccagnella, 2005: The COSMO-LEPS mesoscale ensemble system: Validation of the methodology and verification. Nonlinear Processes Geophys., 12, 527-536, https://doi.org/10.5194/npg-12-527-2005.

_ A. Montani, and T. Paccangnella, 2008: A spatial verification method applied to the evaluation of high-resolution ensemble forecasts. Meteor. Appl., 15, 125-143, https://doi.org/10.1002/met.65.

Mass, C. F., D. Ovens, K. Westrick, and B. A. Colle, 2002: Does increasing horizontal resolution produce more skillful forecasts? Bull. Amer. Meteor. Soc., 83, 407-430, https://doi.org/ 10.1175/1520-0477(2002)083<0407:DIHRPM>2.3.CO;2. 
Matheson, J. E., and R. L. Winkler, 1976: Scoring rules for continuous probability distributions. Manage. Sci., 22, 1087-1095, https://doi.org/10.1287/mnsc.22.10.1087.

Mittermaier, M. P., 2014: A strategy for verifying near-convectionresolving model forecasts at observing sites. Wea. Forecasting, 29, 185-204, https://doi.org/10.1175/WAF-D-12-00075.1.

— , and R. Bullock, 2013: Using MODE to explore the spatial and temporal characteristics of cloud cover forecasts from high-resolution NWP models. Meteor. Appl., 20, 187-196, https://doi.org/10.1002/met.1393.

— , and D. B. Stephenson, 2015: Inherent bounds on forecast accuracy due to observation uncertainty caused by temporal sampling. Mon. Wea. Rev., 143, 4236-4243, https://doi.org/ 10.1175/MWR-D-15-0173.1.

_ , R. North, A. Semple, and R. Bullock, 2016: Feature-based diagnostic evaluation of global NWP forecasts. Mon. Wea. Rev., 144, 3871-3893, https://doi.org/10.1175/MWR-D-15-0167.1.

Montani, A., and Coauthors, 2003: Operational limited-area ensemble forecasts based on the Lokal Modell. ECMWF Newsletter, Vol. 98, 2-7, ECMWF, Reading, United Kingdom, https:// www.ecmwf.int/sites/default/files/elibrary/2003/14626-newsletterno98-summer-2003.pdf.

Murphy, A. H., 1969: On the "ranked probability score." J. Appl. Meteor., 8, 988-989, https://doi.org/10.1175/1520-0450(1969) $008<0988$ :OTPS $>2.0$. CO;2.

Neuwirth, E., 2014: RColorBrewer: ColorBrewer palettes, version, 1.1-2. R package, https://CRAN.R-project.org/ package $=$ RColorBrewer.

Radanovics, S., 2014: Spatially coherent precipitation downscaling with meteorological analogues. Ph.D. thesis, Université de Grenoble-Alpes, Saint-Martin-d'Hères, France, 251 pp., https:// tel.archives-ouvertes.fr/tel-01231000/.

R Core Team, 2016: R: A language and environment for statistical computing. R Foundation for Statistical Computing, https:// www.R-project.org/.

Roberts, N., and H. Lean, 2008: Scale-selective verification of rainfall accumulations from high-resolution forecasts of convective events. Mon. Wea. Rev., 136, 78-97, https://doi.org/ 10.1175/2007MWR2123.1.

Röpnack, A., A. Hense, C. Gebhardt, and D. Majewski, 2013: Bayesian model verification of NWP ensemble forecasts. Mon. Wea. Rev., 141, 375-387, https://doi.org/10.1175/MWR-D-11-00350.1.

Roulston, M. S., and L. A. Smith, 2002: Evaluating probabilistic forecasts using information theory. Mon. Wea. Rev., 130, 1653-1660, https://doi.org/10.1175/1520-0493(2002)130<1653: EPFUIT $>2.0 . \mathrm{CO} ; 2$.

Schwartz, C. S., and R. A. Sobash, 2017: Generating probabilistic forecasts from convection-allowing ensembles using neighborhood approaches: A review and recommendations. Mon. Wea. Rev., 145, 3397-3418, https://doi.org/10.1175/MWR-D-16-0400.1.

- G. S. Romine, K. R. Fossell, R. A. Sobash, and M. L. Weisman, 2017: Toward 1-km ensemble forecasts over large domains. Mon. Wea. Rev., 145, 2943-2969, https://doi.org/ 10.1175/MWR-D-16-0410.1.

Siegert, S., 2017: SpecsVerification: Forecast verification routines for ensemble forecasts of weather and climate, version $0.5-2$. R package, https://CRAN.R-project.org/package $=$ SpecsVerification .

Skinner, P. S., L. J. Wicker, D. M. Wheatley, and K. H. Knopfmeier, 2016: Application of two spatial verification methods to ensemble forecasts of low-level rotation. Wea. Forecasting, 31, 713-735, https://doi.org/10.1175/WAF-D-15-0129.1.

Skok, G., and N. Roberts, 2016: Analysis of fractions skill score properties for random precipitation fields and ECMWF fore- casts. Quart. J. Roy. Meteor. Soc., 142, 2599-2610, https://doi.org/ 10.1002/qj.2849.

South, A., 2011: Rworldmap: A new R package for mapping global data. $R J ., 3,35-43$, http://journal.r-project.org/archive/2011-1/ RJournal_2011-1_South.pdf.

Steinacker, R., C. Häberli, and W. Pöttschacher, 2000: A transparent method for the analysis and quality evaluation of irregularly distributed and noisy observational data. Mon. Wea. Rev., 128, 2303-2316, https://doi.org/10.1175/1520-0493(2000)128<2303: ATMFTA $>2.0 . \mathrm{CO} ; 2$.

— , and Coauthors, 2006: A mesoscale data analysis and downscaling method over complex terrain. Mon. Wea. Rev., 134, 2758-2771, https://doi.org/10.1175/MWR3196.1.

— D. Mayer, and A. Steiner, 2011: Data quality control based on self-consistency. Mon. Wea. Rev., 139, 3974-3991, https:// doi.org/10.1175/MWR-D-10-05024.1.

Termonia, P., D. Degrauwe, and R. Hamdi, 2011: Improving the temporal resolution problem by localized gridpoint nudging in regional weather and climate models. Mon. Wea. Rev., 139, 1292-1304, https://doi.org/10.1175/2010MWR3594.1.

Theis, S. E., A. Hense, and U. Damrath, 2005: Probabilistic precipitation forecasts from a deterministic model: A pragmatic approach. Meteor. Appl., 12, 257-268, https://doi.org/10.1017/ S1350482705001763.

Van Weverberg, K., N. P. van Lipzig, L. Delobbe, and D. Lauwaet, 2010: Sensitivity of quantitative precipitation forecast to soil moisture initialization and microphysics parametrization. Quart. J. Roy. Meteor. Soc., 136, 978-996, https://doi.org/ 10.1002/qj.611.

Vincendon, B., V. Ducrocq, O. Nuissier, and B. Vié, 2011: Perturbation of convection-permitting NWP forecasts for flash-flood ensemble forecasting. Nat. Hazards Earth Syst. Sci., 11, 1529-1544, https://doi.org/10.5194/nhess11-1529-2011.

Wang, Y., S. Tascu, F. Weidle, and K. Schmeisser, 2012: Evaluation of the added value of regional ensemble forecasts on global ensemble forecasts. Wea. Forecasting, 27, 972-987, https://doi.org/ 10.1175/WAF-D-11-00102.1.

Weniger, M., and P. Friederichs, 2016: Using the SAL technique for spatial verification of cloud processes: A sensitivity analysis. J. Appl. Meteor. Climatol., 55, 2091-2108, https://doi.org/ 10.1175/JAMC-D-15-0311.1.

, F. Kapp, and P. Friederichs, 2017: Spatial verification using wavelet transforms: A review. Quart. J. Roy. Meteor. Soc., 143, 120-136, https://doi.org/10.1002/qj.2881.

Wernli, H., M. Paulat, M. Hagen, and C. Frei, 2008: SAL-A novel quality measure for the verification of quantitative precipitation forecasts. Mon. Wea. Rev., 136, 4470-4487, https:// doi.org/10.1175/2008MWR2415.1.

_ C. Hofmann, and M. Zimmer, 2009: Spatial forecast verification methods intercomparison project: Application of the SAL technique. Wea. Forecasting, 24, 1472-1484, https://doi.org/ 10.1175/2009WAF2222271.1.

Wickham, H., 2007: Reshaping data with the reshape package. J. Stat. Software, 21 (12), 1-20, https://doi.org/10.18637/ jss.v021.i12.

, 2009: Ggplot2: Elegant Graphics for Data Analysis. Use R! Series, Springer, 216 pp., https://doi.org/10.1007/978-0-38798141-3.

,2011: The split-apply combine strategy for data analysis. J. Stat. Software, 40 (1), 1-29, https://doi.org/10.18637/jss.v040.i01. 
Wittmann, C., T. Haiden, and A. Kann, 2010: Evaluating multiscale precipitation forecasts using high resolution analysis. Adv. Sci. Res., 4, 89-98, https://doi.org/10.5194/asr-4-89-2010.

Wulfmeyer, V., and Coauthors, 2008: The Convective and Orographically Induced Precipitation Study. Bull. Amer. Meteor. Soc., 89, 1477-1486, https://doi.org/10.1175/1520-0477-89.10.1469.

Yano, J.-I., and B. Jakubiak, 2016: Wavelet-based verification of the quantitative precipitation forecast. Dyn. Atmos. Oceans, 74, 14 29, https://doi.org/10.1016/j.dynatmoce.2016.02.001.

Zappa, M., and Coauthors, 2010: Propagation of uncertainty from observing systems and NWP into hydrological models: COST731 Working Group 2. Atmos. Sci. Lett., 11, 83-91, https://doi.org/ 10.1002/asl.248.
Zepeda-Arce, J., E. Foufoula-Georgiou, and K. K. Droegemeier, 2000: Space-time rainfall organization and its role in validating quantitative precipitation forecasts. J. Geophys. Res., 105, $10129-10146$, https://doi.org/10.1029/ 1999JD901087.

Zimmer, M., 2010: Merkmalsbezogene Verifikation hochaufgelöster Niederschlagsvorhersagen für Deutschland. Ph.D. thesis, Johannes Gutenberg-Universität, Mainz, Germany, 168 pp., https:// d-nb.info/1009714147/34.

, G. C. Craig, C. Keil, and H. Wernli, 2011: Classification of precipitation events with a convective response timescale and their forecasting characteristics. Geophys. Res. Lett., 38, L05802, https://doi.org/10.1029/2010GL046199. 